\title{
Rethinking the Formal Methodology (II): Cognitive Content of Relativity (On the Centenary of General Relativity)
}

\author{
George Kirakosyan \\ Physics Department, State Engineering University, Yerevan, Armenia \\ Email: matgeorg48@gmail.com
}

Received 1 December 2015; accepted 19 March 2016; published 22 March 2016

Copyright @ 2016 by author and Scientific Research Publishing Inc.

This work is licensed under the Creative Commons Attribution International License (CC BY).

http://creativecommons.org/licenses/by/4.0/

(c) (i) Open Access

\begin{abstract}
An attempt to epistemological completion of formal-math theories of relativity is presented. Causal interpretations of SR and GR are suggested. The problem to physical gist of gravity is explained as a contradiction of cognition vs. intuition. Gravity phenomena are represented as unexplored peculiarity of basic particles. The gravity constant is deduced from the known parameters of the electron.
\end{abstract}

Keywords

Einstein, Special Relativity, General Relativity, Gravity Constant

\section{Introduction}

We can't solve problems by using the same kind of thinking, we used when we created them.

Einstein

\subsection{Critical Remarks, Objective and Methodology}

The significance of gravity and relativity theories as well as the tremendous merits of genius Newton, Einstein and other distinguishable classics is undeniable in today's physics. Meanwhile, we shall emphasize that existing works on examined area both significantly famous ones and not so much known ones, mostly remain as formal-mathematical theories, since any of these does not yet answer such natural question as: what is the physical nature of gravity?

The problem requires explanation of causal link between gravity and material substance that needs to be solved to achieve unambiguous clarity on the issue.

How to cite this paper: Kirakosyan, G. (2016) Rethinking the Formal Methodology (II): Cognitive Content of Relativity (On the Centenary of General Relativity). International Journal of Astronomy and Astrophysics, 6, 23-55.

http://dx.doi.org/10.4236/ijaa.2016.61004 
Meanwhile, majority of contemporary theorists consider sufficiently correct quantitative descriptions only as the ultimate goal of the research. Moreover, similar formulation of a problem may sound somewhat unusual-lawless in their view. It seems the deal is not in physicists' competency at all, as they learned to answer the questions "how much is it" before the definition "what it is", within adopted ideology and unspoken standards in modern physics. We urge to draw attention to the important fact in the context:

The same experimentally known gravity constant is used in Newton's gravity as well as in Einstein's GR and in many alternate theories by different variations; by the same, the physical essence of gravity remains an unresolved mystery of nature, as it is unanswered yet where from this constant has arisen and why its value is it?

Thus, the significance of existing theories may be evaluated mostly by the quantitative agreement of their results with observations rather than by the cognitive promotion in the subject area, that indicates a pure technical character of the known attempts and acting criteria. The problem is basically important and long disputable, to begin without certain presentation. It demands some detailed examination of a long passed way that has brought to the formation of current ideology, methodology and to a certain stalemate in problematic sections of physics in general. Mentioned intention can hardly be attractive to nowadays' theorists; we realize that it might look as an anachronism, as something long withdrawn from practice because of previous attempts. Meanwhile, it is just inevitable in stated task, since investigated questions demand conceptual-epistemological analysis more than technical. Therefore, certain time and good-enough patience of readers are required for mastering new concepts and language which are actually well forgotten old ones. We start with registration of few important guiding points. As mentioned above:

a) The theoretical studies of phenomena are equalized to their quantitative investigations in modern physics, adopted by some historical circumstances (math modeling of reality)

The problem was widely discussed long ago among distinguished coryphées of physics, as well as philosophers. The question has mostly related to revealing new-unusual quantitative properties and relations peculiar to the elementary particles of substance (quantum relations). The final key principles and the "correct" methodology were adopted through hard disputes, by the decision of majority despite the unanswered questions. Readers can find some detailed criticism and principles that we will follow in Ref. [1].

We shall emphasize the absence of author's intention to announce existing theories and achieved results on the subject as "something wrong at all". Nevertheless, we have seen:

b) The objective of this work is the cause-cognitive interpretation and completion of the studied subject that is investigated experimentally and mainly quantitatively (mathematically), thanks to deserving researcherspioneers

It must give cognitive "body and blood" to formal math theories, transforming these to conceptually complete, real-physical ones, by author's intention. By comprehensible logic, a critical overview of studied object is necessary for such expectation. The subject of methodology is too large to discuss it fully. We can suggest also Ref. [2] (Russian) on the issue. We have seen a nice book of L. Brillouin as the most valuable on the subject Ref. [3]. We bring also his wonderful words on significance of criticism and reexamination of views in science; "Fanatical veneration of any theory is incorrect-they are improving!" Thus, the possible representation of the presented work as a kind of "encroachment" of deserving names and their merits will be deeply unfair. Meanwhile, we just need to agree that any authoritative scientist, even with great merits, may be an ordinary man only, uninsured of human mistakes and misconceptions. The development of science has never gone smooth and straight ahead. Nevertheless, researchers often have been forced to return to the rejected ideas, correcting their mistakes. Then we can assert:

The periodical overview of passed way in scientific research must be permissible and necessary; otherwise, we may get a confessional doctrine-instead of realistic science.

Coming to a), we need to explain our approach to the mentioned designation of physical theory in general, to be clear what we are doing next. The matter is we are forced to overpass the adopted hard instructions and recipes on the methodology of physics to get some new opportunities in our investigations. The lacks of accepted paradigm of physical science was discussed and has been criticized by authoritative scientists particularly by Einstein. Therefore, we can be extremely short. The adopted approach is considered as a sufficient condition for complete representation and study of reality, in dominant present ideology. Below unspoken opportunities are supposed by the same.

c) We have the ability of revealing the actual picture of reality through experiments and observations only 


\section{d) Our abstract-quantitative descriptions completely correspond to reality}

Thus, the task of finding "enough-correct" descriptions of "real facts", revealed by experiments, seems as the final objective of a good physical theory. The implementation of further, increasingly complicating experiments and creation of a huge system of quantitative descriptions, covering as many possible facts and phenomena are seen as the desirable task of physical science within conformity of presented paradigm a), c), d).

We briefly have depicted above the essence of the adopted formal-math methodology in nowadays physics. Meanwhile, above-described designation of physical theory a) and convictions c), d) can be evaluated as an expression of trivial desire "to simplify works" that, however, causes serious misconceptions and unsolvable situations in result that we are facing today. Some consecutive analysis should be enough to get convinced in above-said. We will examine one bright historical example somewhat related to gravity problem.

The known historical offer of genius Copernicus, on replacement of the geocentric system to a heliocentric, with consequent huge advance in celestial mechanics directly demonstrates the injustice of above presented perception and nowadays paradigm of physical science at all. Let us remember some details on the issue. The early observations of planets and collected data on their movements did not give opportunity to researchers to see any principle in their intricate paths. Let us assume that our ancestors had been satisfied with observed data and they had registered-systemized these as "real laws of nature", in conformity to c). It is easy to comprehend that they would be forced to use certain tremendous-sophisticated system of quantitative description of planets' movement, well conforming to observations, having no idea of the causal essence of phenomena at all. Thus, their "celestial mechanics" would look as some analog of nowadays quantum theory; there would be well tested, working formulas (instructions, tables, diagrams etc.) and full absence of any causal explanation: why does this group of phenomena go namely so?

Copernicus's incredible merit lies in his thought operations-logical judgments that gave a wonderful opportunity to reveal a universal rule in planets' movement that had been observed before as separately different. He has placed an imaginary observer on the Sun and has defined how planets' movements would seem from there. Then, it became possible "to see" imaginary picture of decent paths of planets and the unique rule of their movements, thanks to this judgments. The discussion is about Kepler's laws somewhat generalizing planets' movement, providing their universal description. Newton's law of universal gravity had next huge advance that was obtained from Kepler's laws by technical-mathematical way only (using differential calculus), in a form of short-compact quantitative relation serving as a basis of classic celestial mechanics. There are many examples in classical physics on significance and inevitable necessity of logical operations. Thus, we can emphasize the important fact:

e) The significant progress in physics was achieved thanks to implementation of logical-judging operations, intermediately between experiments-observations and quantitative investigations-descriptions of phenomena

From this and other similar examples, we can remark next obvious conclusion:

f) Mostly, we have no possibility to direct observe the actual values and "the right picture" of reality that we trust and accept as "basic law of nature"

As we saw in above example, we have no capability of direct observation of the regular-beautiful paths of planets around the Sun, and we accepted their existence thanks to our thought operations and applicabilityproductivity of created imaginary picture-model. Our next predictions and calculations are based on the created model and adopted principles and can be confirmed with new observations in some favorable cases. However, we can also comprehend the absence of opportunity of new experiments-observations that may confirm our predictions and theories at all, conditioned by different unsolvable technical restrictions mainly. Then we can only be satisfied with the trusted model due to its completion, until new facts force us review our beliefs. It is the normal-natural way of the development of science by its long history. Thus, we can state trivial simplifications in the declared paradigm a), c) and imperfection of adopted methodology in result.

We have used definitely distinguished concept and approach to significance and methodology of physical science with considering the above presented criticism and guiding principles. Reader must overcome natural skepticism and unfavorable heavy suspicions to applied approach created due to long historic circumstances. It is the price to get some clarity on the studied subject that has been natural in many similar cases. Therefore, reader's own decision is required here to judge how much trust the presented work inspires, and how much useful it seems.

The opportunity of interpreting quantum phenomena and microcosm in whole with implementation of imaginary-figurative representations and universal cause-effect laws of nature are presented in Refs. [1] [4]. We mark 
common principles of applied methodology and deep correspondence of basic assumptions. Many realistic thinkers repeatedly have called to return back to natural way of thinking and to cause-effect interpretations vs. adopted formal methodology as Refs. [5] [6]. However, official viewpoint on the key principles remains long unshakable despite formal declarations "to involve new ideas". Author's approach to the significance of physical science may be expressed in words of wonderful physicist's R. Lindsay who saw the designation of science "comprehension of the essence of things by thinking" Ref. [7] that does not correspond to dominant ideology.

\subsection{Mathematics and Epistemology}

The necessity to clarify the meaning and significance of used concepts and actions to build our science follow from the above outlined approach. The contemporary physical theories may be characterized as quantitative judgments, corresponding to the experimentally established results, as noted above. Therefore, the examination of assumption (1.1. d)) becomes most important as one of the basic criteria of significance of adopted paradigm of physical theory at all. The history of development and abstract-generalized character of math apparatus are known to us from school education that we formulate as below:

We evaluate mathematics as a wonderful human creation, serving as a special language and rational tool, providing important opportunity for description and investigation of kinds of quantitative relations inherent to studied subjects.

By definition, mathematics must work under certain logical control as a "language-tool"; it cannot work by itself and always be useful to us, because of the possibility of its unclaimed applications as with any other tools. It means researcher-operator must well comprehend the meaning and clear target of math actions-operations to get somewhat guaranteed-valuable results. We are forced to mention that modern physicists are often guided by pure formal-mathematical demands only, in abstraction from actual peculiarities of studied real physical objects in dominant practice. They are inclined to trust the strong math rules only due to their standard education, looking at the ordinary logic arguments as some "not enough clear things", therefore, as undesirable and not mandatory! The ignorance of logical control in math operations leaves only the way of quantitative considerations by trivial test-error principle, which increases their works and minimizes the productivity, especially in complicated cases. Meanwhile, a careful examination of the mutual relation of mathematical and logical rules may clarify their common roots and groundlessness of seeming contradictions among these. We can be convinced from history in the incomparable success of mathematics as well as of natural sciences in general, namely, in the period when logical and quantitative considerations were applied with organic combinations. As is known, different kinds of quantitative operations and math functions may be reduced to basic concepts of unit \& null (1\& 0$)$ and to application of abstract quantity conservation law $(1=1 \neq 0)$. It reflects the general principle of nature: a real "thing" can be created from another real one only and cannot be transformed to "nothing". Thus, all kinds of math equations and operations are based, and these may be reduced to above-mentioned elementary concepts and simple actions (binary numeral system and modern computing technology may serve as simple evidences to above-said). We will remark for our guidance:

a) Mathematics is a specialized section in common study system developed as generalized-abstract, separate discipline by using special-rational symbolism. The coincidence and correspondence of mathematics with the reality and its workability are conditioned by the implementation of the quantitative conservation laws, reflecting universal peculiarity of phenomena in nature. The quantity conservation laws themselves are based on cause-effect strong logic. Thus, mathematics may be linked to natural science with clear initial definitions the real objects and their properties, expressed by math concepts and symbols

It is easy to realize the above, taking into consideration that the same formulas and equations can be used in different cases, depending what meaning is attributed to symbols.

It follows from mentioned:

b) Neither the logical considerations themselves nor pure math methods can serve us independently and sufficiently to complete descriptions of real phenomena. Their clear linked application only can serve as a possible effective analytical way of research

Meantime, above-said is not some discovery in scientific methodology; our ancestors actually had long worked by the same principle. The matter is early thinkers had insufficient math knowledge (as well as experimental capabilities). They somewhat have ignored their significance at all, as natural, being guided by logical judgments mostly. 
The unprecedented shift has occurred thanks to the opening of Newton-Leibniz differential calculus. Physicists have decided to review previous methods at all, leaning on pure math methods only, ignoring logical judgments as "something traditional-ineffective", being deeply impressed with the unprecedented success and seeming capabilities of new methodology! The short-term valuable results (the known success of quantum representations) had been perceived as weighty confirmation of reformers' decision, in favor of involved formal-math methodology. Unprecedented problems and confusions however, have arisen with the time because of innovation as Ref. [6]. Thus, we can observe from above-said:

c) The Ideology and methodology in physics resolutely deflected from one incomplete-ineffective to another extreme, due to historic circumstances ${ }^{1}$

We have shown above that logical considerations are mostly ignored in present formal methodology. To be more precise, they are actually used spontaneously, in some silent-arbitrary manner, because the attempts to build "pure mathematical physics", without any logical considerations at all, obviously, will be an extreme abstraction that can hardly have any significance! Then simple follows the next unexpected and important demand:

d) Logical considerations in natural science must be used either on the sufficient systematized basis-or be excluded at all

The second way will be obviously speculative and can hardly be useful for someone. Then there remains no choice other than first. Based on above-said we accept:

e) The experimental results, logical and quantitative considerations must be adopted as mandatory components in the complete methodology of realistic natural science

Thus, one of mentioned three basic tools is ignored in disputable sections of modern physics in fact, due to various historic circumstances. The question has been long discussed by many distinguished coryphées and we tried to present briefly the whole importance of the problem to revise used methodology. The reader, himself, may judge the opportunities and productivity of suggested application by following content. We will mention another important fact on this as well:

f) The combination of logic and quantitative considerations provides a new important tool of research, putting necessary restrictions on each other and mutually controlling both applications

The borders and limitations of applications in study process become clear and appear themselves in natural ways with e) deriving from properties and peculiarities of real objects (which is one of the main problems in present formal-math methodology!). It significantly increases research capabilities and decreases unnecessary mathematisation of problems. The simple examples may demonstrate meaning and rightness to the above-said.

Let's mark apples quantity as $A$, and number of children $B$. We use operation $A / B$ and not $B / A$ to distribute apples to children, despite the two operations are equally lawful from formal math's point (as a definition ratio of two numbers). The matter is, here we silently considered that $A$ may be fractional and $B$ never can, i.e., 1) we have applied logical restriction, which frees us from examination of second operation); 2) we get two contrary results $\pm A$ when we define the radius of circle with known surface. We choose $+A$, ignoring $-A$, because we do not use in practice the circle with minus radius (logical decision); 3) we ignore the sizes of two cities when we speak about their distance. We use such approximations to simplify our work, clearly realizing their restriction and relative significance (idealized, thought operations). These simplest examples demonstrate whole triviality of the supposed opportunity (1.1. d)) and the necessity of initial consideration of physical peculiarities of real objects, parallel with the quantitative operations. It provides the necessary conditions and important instructions to quantitative operations in the researches. Mentioned demands were mostly regarded in classical physics in natural ways (without special declaration), and are mostly ignored in formal methodology due to formed circumstances (also without declaration!). The abstract math concepts become confused with the real-physical ones in the research works that created artificial problems and aroused unsolvable paradoxes in consequence.

The clearly formulated demand to divide physical and math concepts from each other reader can find in Ref. [3] (relating to observation frames, particularly). Einstein has resolutely demanded in his disputes to build physical theories on the conceptual basis, and, the used concepts to connect with the real objects as Ref. [8] that we see in the same context. Mentioned demands however, have met a hard criticism and decisive resistance of majority of theorists and physics has deviated to a present formalism as a result. We shall rely on the ideas and demands of undeniable founders of physics on methodology that we see unfairly rejected.

Outlined remarks and approaches serve us in further examination of study subject.

${ }^{1}$ It may to illustrate division of physics on Classical and Postclassical different ideologies. 


\section{Physical and Cognitive Significance of SR}

Small is the number of people who see with their eyes and think with their minds.

Einstein

\section{Causal Interpretation to SR}

GR provides certain amendments to Newton's gravity, mostly confirmed experimentally, pointing on its significance and superiority. Meanwhile, logical problems related to unknown physical essence of Newton's gravity were aggravated more with introduction of new unclear categories. These have risen from linking SR to gravity. We shall notice certain improvidence in Einstein's initial approaches with cognitive viewpoint that characterizes the present formal methodology in general and plays a key role in further conclusions.

The problem lies in usage of cognitively uncertain concepts to develop new theories. As is known, SR contains some unresolved logical clouds and paradoxes remaining as subjects of hard disputes at present. It is possible to comprehend however, that involvement of unclear categories for solving current problems may complicate them much more by adding an unexplained object to the other dark one (long-term problems with relativity theories evidence it!). Thus, we need to clarify the cognitive meaning of SR before examining GR. We begin with examination of known disputable questions, supposing reader's acquaintance with the subject.

\section{1) Twins' paradox}

Travelling brother remains younger in his spacecraft on relation to homebody because he undergoing accelerations that puts certain asymmetry in their conditions; it looks enough-basic to assertion that namely traveler will remain young in relation to homebody, within accepted SR interpretation. However, next simplest objection is possible. We can put symmetry in the experiment by using triplet of brothers for example. One of them can stay in home and two others we will send to travel on contrary directions. Then, it becomes impossible to preferring one of situation for traveling brothers and to decide someway who of them will be old or young? Each of them can calculate by SR principles, using Lorentz transformations (LT), concluding that his brother remains young and not he! Paying attention the viewpoint of homebody also, we fall into deepest confusion as becomes just impossible to find any decision that may be common-acceptable to everybody. It is one of basic criteria to objectivity of science to which SR is not corresponds by its present interpretation as shown above. Similar subjectivism and logic objections are much that pushes many thinkers to reject the significance of SR at all despite some its results are used in engineering level (as $E=m c^{2}$ ). Described reality demands some not trivial approach, does not sacrificing kind of arguments in a favor to others as it takes place in adopted interpretation of SR. We will look some new examples as well, without quantitative operations, demanding clear answers and giving some hint by the same-where need to look for the causal explanations to logic questions.

\section{2) Change of time and length units with movement}

The astronomers had known about velocity of light long-before SR and they well realized that observed picture of any far object corresponds to some of its early state that may be significantly different from the actual one. Then we can comprehend that our brother-twin in the far planet will seem young to us same as he will see us because of limited speed of light. There is nothing mystical here; we can realize that a certain time is needed for light to reach us, which simply explains the phenomenon of observable time difference on distance. Let us imagine someone who travels from our place to our brother. We can realize that in the end of the way his watch will correspond to the brother's watch and his life. Then, it is possible to conclude that his watch will look like "slowed down" during movement to "compensate" seeming difference of time! The seeming correlation and "dependence of time on speed" of traveler becomes clear with above-said; the observable course of traveler's time must slow down more with increased speed of his movement from us. We will also see some distortion in the length of things in movement process, in correlation to speed. We see the two ends of moving meter not at the same moment because of limited speed of light; therefore, its length will look distorted to us, depending on velocity and direction of its movement. We can conclude also that all above judgments are symmetrical and reversible for brothers: particularly, if the traveler moves to us from brother, then his watch will look accelerated to us, and it will seem opposite to brother ${ }^{2}$. We can see nothing against logic in these thought experiments and unusual conclusions as we realize well that the discussion refers to observed values and not to actual ones,

\footnotetext{
${ }^{2}$ The change of time course must be observed during of travel "to compensate the time difference" and to reach of "brother's time". Physicists can realize that Doppler Effect only plays role here.
} 
which can be different at all. Readers have the right to ask a question here: what do we mean under term of "actual values" in this case? We find the answer from (1.1. f)) in analogy to planets' orbits and Copernicus' wonderful lesson. As we saw, he had used the imaginary observation frame and he took the imaginary picture of orbits as "actual".

Thus, we can accept as "actual" the values and imaginary picture of phenomenon that may be observed (measured) if we will be able to realize instant measurements. It sounds very strange, of course, as we will never be able to see such reality! However, we can create it through our judgments and calculations. We have the same right do it and to trust our conclusions, as we unequivocally believe in the existence of decent orbits of planets in present time. We often use similar actions in classical physics actually, when we equalize friction forces to zero in some cases, for example, well realizing that we cannot do it at all, as it is only an idealized representation (1.2.3.). We just need to take care that we see phenomena not instantly as we silently have accepted (i.e., we cannot take the velocity of light as infinite, same as friction in zero). To get the actual picture of examined phenomena using necessary corrections, we need to remember; the reason of distortions (errors) is the limited speed of light, thanks to which we see "time difference on the distance" and "dependencies of units of time and meter on speed". Then, it becomes simply clear that "the time difference on distance" actually is not depending on speed or on the form of the way but on distance only. We can suppose that the traveler moves by different routes and variable speeds, even exceeding the velocity of light; the ultimate result will be the same because:

a) The actual factors causing SR phenomena are the velocity of light and the distance to observed point. Thus, it becomes unimportant how to reach there ${ }^{3}$.

Let us imagine now a researcher who does not realize the reason and physical essence of described phenomena. Then he observes and opens "changes" of time course and length of things in parallel (in correlation) to the speed of movement and he interprets these as the "real laws of nature, revealed by experiments" (1.1. c). Then he declares; "the speed of movement causes the actual changes of physical values"! He makes some quantitative judgments also and discovers certain formulas, corresponding to the results of his observation defining the "actual dependencies of physical values on speed".

Meantime, he faces some logical complications with adopted representations (that we can comprehend already from above content!). Particularly, he cannot answer clearly, what will happen to the traveler if he will move faster than the velocity of light? Then he supposes-declares for such cases especially (to be free from next huge complications): "the velocity of light is maximal in nature that cannot be exceeded any way!" Meanwhile, he has deeply changed the meaning of the basic concept of "speed" by the same. The matter is:

b) The concept of "speed" is related to two objects, thus, it can be only relative. Meanwhile, in SR it silently acquires some independent-absolute significance

Our researcher faces many similar curious questions and he does not find any explanation: why things must be so contradictory to logic. Then, he decides to "close his eyes" on the logical arguments as "some non mandatory things in physics". Meantime, his theory works successfully despite many logical flaws, since quantitative results mostly correspond to observations that look as weighty evidence to its rightness! Thus, we have suggested above a cause-logical explanation to essence of SR that we continue to examine further.

3) Mechanical speed, light velocity and "space-time"

One historical reason to introduce LT and create SR is linked to measurements of the velocity of light in relation to hypothetical environment "ether" that was profoundly different from the expected ones (see Michelson-Morley Experiments.) It was confirmed:

c) The measured velocity of light is invariant, independent from the movement of the observer and the source of light relative to each other

This result contradicts to Galileo's relativity principle (GRP) in fact, as it breaks the rule of speeds' summation on which Newton's mechanics and classical physics are based. We will examine below one extremely simple thought experiment that demonstrates the rightness of mentioned allegation, demanding its clear explanation, not allowing a cover-up of the question with math manipulations.

Let us suppose the experimenter defines the velocity of platform using some standard gun, fixed on it, by measurements of bullet velocity (Figure 1).

The velocity of bullet $V$ is known initially. Its measured value will be $V_{m}=V+V_{x}$ and speed of the platform may be defined as $V_{x}=V_{m}-V$, according to GRP and summation rule of speeds. We need to notice initially that the same result is possible to confirm by direct measurements of platform speed, without using the gun and

${ }^{3}$ It relates to a summary final result of phenomenon. 


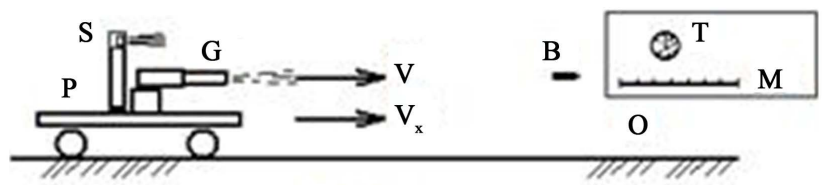

Figure 1. P: platform; O: observer; B: bullet; V: speed of bullet; $\mathrm{V}_{\mathrm{x}}$ : speed of platform; G: the gun; S: the source of light signals; T, M: the clock \& meter.

summation rule of speeds. Such opportunity and coincidence of two measurements allow us to adopt abovepresented result as correct, corresponding to reality. Thus, we can accept GRP as doubtless, and as a base to other judgments in virtue of it. The creators of SR, also well comprehending its fundamental significance, have announced the correspondence of SR to GRP. Mentioned assertion however, actually remains a verbal declaration only, containing deep internal contradictions that we will show further.

Let's suppose now the observer has used the light source $S$ together with gun. He measures light velocity and got $V_{m}=c$, according to some experiments and to basic principle of SR. He got $V_{x}=0$ if he used GRP and the same summation rule. Then, he decided to follow SR exclusively, as this result does not coincide to reality and it obviously is wrong. The SR formula of speeds summation, corresponding to condition of experiment, is below:

$$
U=\frac{U^{I}+V}{1+\frac{U^{I} V}{c^{2}}}
$$

Applying in (2.1) the conditions of experiment $U=c, V=c, U^{I}=V_{x}$ we get $V_{x}+c=V_{x}+c$ that shows the problem is irresolvable, as $V_{x}$ may have arbitrary values. Moreover, the second experiment does not allow us to tell whether the platform is moving or no! Thus, we can surely mark:

The light signal cannot replace a bullet and play the same function of the tool of measurement, independent of our initial convictions or used interpretations.

This fact shows certain qualitative difference of the velocity of light and mechanical movement that actually breaks GRP. Thus, we need an answer to an important question:

d) What is the difference of the velocity of light and bullet movement that does not allow their replacement? Meantime, it is only the first part of the problem related to the velocity of light. There is a second important question too that we have formulated below:

e) How the velocity of light becomes invariant, independent of relative movement of the source \& observer that directly contradicts to the known rule of summation of speeds?

We must exhaustively answer the questions d) and e) to comprehend the cognitive meaning and physical essence of SR. As we see from (2.1.1) and (2.1.2), SR actually offers below interpretation to the relation (2.1). A new hypothetical participator is actually supposed-introduced in the studied phenomena by linking the "time" to "coordinates" as the "space-time", attributing hypothetical properties to it "to change the physical values with relative speed" as it is necessary for explanation of the observed results. Thus:

f) SR actually considers the inevitable errors of measurements, arising from restricted and constant velocity of light that we are forced to use as a tool of study. Mentioned corrections however, are in fact attributed (verbally) to a cognitively uncertain hypothetical category "space-time" that is represented as an independently existing reality by the virtue of supposition of its own properties "influencing the real physical objects". We showed (2.1.2) the non-necessity of attributing unexplainable properties to our measuring tools (clocks \& meters) "to change their values" with mystical-subjective dependence on relative speed. Thus, the introduction of the concept of "space-time", as a kind of unity "carrying own properties" may be evaluated as a free creativity (inputting a hypothetical reality) that causes explainable logical confusions. Thus:

g) It is important to evaluate the false-fictional gist of "space-time" to remove it from physics, as a directly obstructive factor in problematic subjects related to it

Question concerns to gravity problem in first place, after SR, where "space-time" plays a key role, as well as to physics of elementary particles, where it is involved under the modified name "physical vacuum", with new additional "properties-obligations", necessary for "explanation" of phenomena in this complicated disputable area. 
The speculative essence of "space-time", as some kind of independently existing "unobservable reality", may be easily comprehensible if we only agree to take into consideration the obvious-undeniable facts. As we can be convinced, the single natural constant c only quantitatively characterizes full spectrum properties of "space-time" what we actually see in SR formulas ${ }^{4}$. Thus, the whole significance of "space-time" can be reduced to the consideration of the velocity of light in our measurements as it is done in SR in fact; and we already have an exhaustive answer to question: why we must consider light velocity in our formulas (2.1.3. f)).

We bring one additional argument also on the false essence of "space-time". The known combination of three-dimensional coordinates (i.e. the volume) we meant initially under the term of "space". Thus, next realistic question becomes lawful: "about coordinates of what real things are we talking?". The same is right for the concept of "time" too, as we cannot define the "time" (its course, or the interval between some regular events) without using corresponding material objects. These judgments show that:

h) The concepts of "space" as well as "time" can be comprehended as the attributes-properties of real material objects; these cannot have physical meaning by themselves-separately, as well as in some of their combination (same as, the concept of "speed" does not have meaning by itself without pointing the objects it relates). We need to mention for justice that Einstein had noticed the meaninglessness of the concept of "space" separately from material objects as in Ref. [9]. This judgments and remarks show that the famous innovation of H. Minkowski combination of "space-time" has neither cognitive nor physical significance, if we wish to keep initial meanings of used terms. It will be an obvious nonsense to say; "some kind of combination of properties has its own properties" even from morphological viewpoint. Thus, the concept of "space-time", without mentioning material objects these belong, may have only verbal psychological significance. It creates the psychological impression only to remove the necessity of experimental confirmation of the reality of Lorentz "ether" that was demanded with its definition ${ }^{5}$. The "space-time" has brought a whole group of cognitive mysteries with him as well, on which several generations of thinkers have been working untiringly! The "ether" however, silently continues functioning under new name, because the question: how physical values vary with relative movement needs an answer, same as before. The mentioned fact pushes many researchers to attempt to recover the forgotten "unobservable ether" in modern physics, as the "space-time" plays the same role in its actual interpretation.

\section{4) Problems with dual character of light velocity}

As shown above, the introduction of LT and "universal ether" that actually was replaced with the "spacetime" in SR, were conditioned by properties of light as kind of physical reality ("el.mag field" in generalized name) that plays some important role in our measurements and in our world in whole. Our problems with light velocity and its difference from mechanical movement we have divided on two, (2.1.3. f)) that are its restriction $\&$ invariance as mentioned in stated questions (2.1.3. b)) and (2.1.3. e)) as well. We will pay attention first on the certain difference of light speed from mechanical. We know that "mechanical speed" relates to a two objects equally and symmetrically; i.e. "mechanical speed" may be defined as the common property of two objects. Meanwhile, light velocity may be defined by different ways: as an individual or, own attribute of el.mag field's exclusively, in first. It is the wave propagation velocity, defined by own parameters of field only:

$$
V_{P}=\lambda / T=\lambda v=\text { const }=c
$$

where: $\lambda, T, v$ are wavelength, the period and frequency of light accordingly. (2.2) calls also phase velocity. Thus, we can emphasize that $V_{P}$ is the exclusively own wave character of field that deeply different of "mechanical speed" by the same ${ }^{6}$. The velocity of energy transfer by wave group is accepted as the second definition of wave velocity that corresponds to a classical movement of particle; it calls also wave group velocity:

$$
V_{G}=L / t
$$

where: $L, t$, are the distance and measured time of wave group's motion, accordingly.

We need to emphasize that in second case the etalons of length and time i.e. the used tools of measurements, are independent; these are not defined by own parameters of field as in first case, and these are introduced externally. Mentioned circumstance is important to answer how the invariance problem of light velocity arose. We know that values of both definitions of light velocity coincide for the vacuum whereas wave dispersion is absent.

\footnotetext{
${ }^{4}$ Academician $\boldsymbol{A}$. Logunov pointed on this obvious fact in his disputes with academician $\boldsymbol{V}$. Ginsburg.

${ }^{5}$ The verbal replacement the "ether" with "space-time" can have only psychological significance, as basic formulas (LT) remain the same.

${ }^{6}$ Mechanical movement presented as a "point" movement that impossible does with the wave in principle, as example.
} 


$$
V_{P}=V_{G}=c
$$

The same significance of two concepts of light velocity at all is adopted usually for the vacuum, due of equality (2.4). We invite to attention however, that mentioned equality is correct within absence of relative movement between wave source and observer; it becomes impaired in their relative movement. This allegation is easy provable in desire. We just need to consider the obvious fact that Maxwell's equations describe el.mag wave behavior only. Thus, LT for the Maxwell equations have generalized these for the waves only whereas wave source and observer' frame are moving in relation each other. As we see, the application of LT for the Equation (2.2) becomes reduced to transformation:

$$
c^{I}=\frac{\lambda^{I}}{T^{I}}=\frac{\gamma \lambda}{\gamma T}=\frac{\lambda}{T}=c
$$

where: $\gamma=\left(1-v^{2} / c^{2}\right)^{0.5}$ is the universal coefficient of LT. As we can see in representations of ST as Ref. [10] and Ref. [11] $\gamma$ relates only to Maxwell's equations by fact, because of LT are proven based on the wave Equations (Maxwell-Hertz equations). The matter is $\lambda \& T$ in (2.5) are own parameters of wave field that become changed for the observer due of relative movement in same significance; their relation remains invariant in observer's frame due of it. Similar changes take place of field force-vector parameters also as example:

$$
\boldsymbol{E}^{I} \boldsymbol{B}^{I}=\boldsymbol{E} \boldsymbol{B} \text {, and: } \boldsymbol{H}^{I} \boldsymbol{D}^{I}=\boldsymbol{H D}
$$

Based on (2.6) and definition of rotor in Cartesian frame from Maxwell's first equation in differential form we can write:

$$
\operatorname{rot} \boldsymbol{E}=\left|\begin{array}{ccc}
i & j & k \\
\frac{\partial}{\mathrm{d} x} & \frac{\partial}{\mathrm{d} y} & \frac{\partial}{\mathrm{d} z} \\
E_{x} & E_{y} & E_{z}
\end{array}\right|
$$

Or,

$$
\operatorname{rot} \boldsymbol{E}=-\frac{1}{c} \frac{\partial \boldsymbol{B}}{\partial t}
$$

And we see:

$$
c=-\frac{1}{\operatorname{rot} \boldsymbol{E}} \cdot \frac{\partial \boldsymbol{B}}{\partial t}=-\frac{1}{\operatorname{rot} \boldsymbol{E}^{I}} \cdot \frac{\partial \boldsymbol{B}^{I}}{\partial t}=\text { const }
$$

(2.7), (2.9) show: light propagation velocity is a natural constant, that is defined by spatial and time changes of force vectors of field; it is exclusively an own peculiarity of the el. mag. field. Then it becomes comprehensible the possibility of expression and detection of this constant in different phenomena connected to el. magnetic field and wave. Thus, the groundlessness of attribution LT to mechanical movement becomes obvious. We must notice that the same explanation to invariance of wave propagation velocity (2.5) actually is contained in Ref. [3], where pointed on "wrongness to take in consideration the changes of wavelength and its period (frequency) together, same time". We see useful to mark also that constant propagation velocity is peculiar not only to el. magnetic waves, and it takes place for mechanical waves too, in particular case, namely when wave source moves in relation to observer. Mentioned asymmetry arises because of participation in phenomenon the environment (that absence for el. magnetic waves). Thus, we can comprehend the lawless of replacement in our experiment the gun with light source (2.1.3. c)) if we take in consideration the wave properties of light (that accepted in most known experiments).

Described explanation is elementary provable in frame of Galilean relativity and classical physics concepts without inputting of hypotheses "changing time course and meters with movement". It is possible to illustrate creation of LT as consequence of confusion and misinterpretation because of we use wave group velocity (2.3) in place of wave propagation velocity of light (2.2). Above-said easy comprehend if use the "particle" properties of light in place of wave parameters and properties. Then we can measure the energy or the impulse of light photons and define velocity of light source, comparing measured values with their initial values in rest condition 
of the object, as example. We can detect exact coincidence of different experimental results, using light signals and the gun, to define platform's speed in the examined experiment, and not necessity of a hypothetical "space-time", by the same. We use one more example to demonstrate how LT may arise as result of confusion two kinds of light velocity. We need forget initially about "difference of time course", "change meters with movement", as well as artificial problems of "clocks synchronization" etc. that arise with SR in consequence of generalization of invariance principle light velocity in relation to mechanical movement also. Thus, we will remain exclusively in frame of GRP and classical physics in our judgments, by the same. We examine next though experiment to demonstrate above-mentioned opportunity (Figure 2).

We suppose necessary conditions in experiment allowing ignore the signals length and its action time to relation of measured values; it means we can look light signal as a moving point-particle. The fixed time by timer in case of rest condition of the rod, will be:

$$
t_{0}=l / c+l / c=2 l / c
$$

We define the time of reaching the signal to right end (1-2) in moving condition of the rod:

$$
t_{R}^{I}=l / c+\Delta l_{1}=l / c+V t_{R}^{I} / c
$$

Using (2.10), we get:

$$
t_{R}^{I}=t_{0} / 2(1-V / c)
$$

We define the time to reach signal back left to timer (2-3) by same judgment:

$$
t_{L}^{I}=l / c-\Delta l_{2} / c=l / c-V t_{L}^{I} / c \text {, and: } t_{L}^{I}=t_{0} / 2(1+V / c)
$$

Summary time to passing signal "ahead and back" of the moving rod will be:

$$
t^{I}=t_{R}^{I}+t_{L}^{I}=t_{0} / 2(1-V / c)+t_{0} / 2(1+V / c)=t_{0} /\left(1-V^{2} / c^{2}\right)
$$

Thus, the relation of two measurements will be:

$$
t^{I} / t_{0}=1 /\left(1-V^{2} / c^{2}\right)
$$

It shows that measured average value of light signal velocity on directions "ahead \& back", (by using "particle" properties of light) in moving frame to relation of light source, will some less compared to its constant wave propagation velocity by the certain factor:

$$
1-V^{2} / c^{2}
$$

The matter is light signal passes long way on the right (1-2) with low speed (c-V) and short on left (2-3) with high speed $(c+V)$. The average sum of time becomes more with the same, than it has in rest condition of the rod. It corresponds to some slowing of light velocity in moving frame, as (2.15). Let us remember that in experiments prior to ST (Michelson-Morley exp. etc) the light velocity was considered mainly as average of two opposite directions; it are noticed in Ref. [3] as well. Such experiments on direct measurements light velocity (Figure 2) in enough accuracy, as well as on the one direction, actually not implemented (the corresponding links author no succeeded to find). Therefore, these cannot be excluded; we hope it can be implemented on future. Meanwhile, the necessity to explanation of deviation (2.15) from GRP and classical physics has arisen due confusion of mentioned two concepts to light velocity as it shown above. As we know already, problem actually

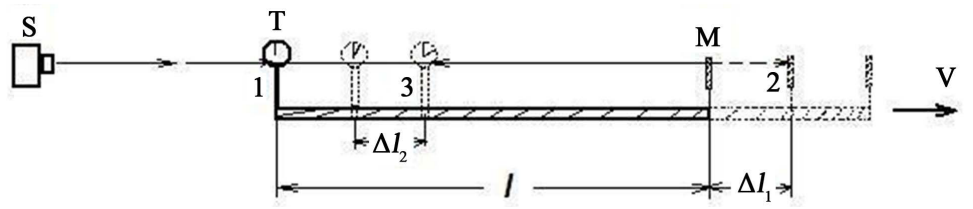

Figure 2. S: Light impulse source; T: Timer with photo sencore; 1: The rod; M: The mirror. The task of experiment is to define light velocity in moving frame by direct measurements time of passage light pulses of the rod with the length $-l$. Timer-T starts work when light hits to it from left side of drawing (point 1) and it stops when light hits from right side (3), after reflection (point 2) from mirror: $\mathrm{M}$, fixed in right end of the rod. 
was "resolved" in SR by attributing to our measuring units "time" and "length" the mystical properties "to changing their values with relative speed", in equally, by half for the each! We can to represent (2.15) as below, agreeing with above interpretation:

$$
1-V^{2} / c^{2}=\gamma_{L} \gamma_{T}=\gamma^{2}, \text { where: } \gamma_{L}=\gamma_{T}=\gamma=\sqrt{1-\frac{V^{2}}{c^{2}}}
$$

$\gamma_{L}=\gamma_{T}=\gamma$ is the known universal factor to "time" and "length" transformation in LT.

Thus, the appearance of LT and "space-time" are possible to explain as consequence of misinterpretation, without going out from classical physics and a new hypothesis, if we review our long-term convictions, which is firstly a psychological problem.

We can use the opposite judgments also to show that the whole significance of LT may be reduced to consideration the factors of light velocity and distance in observations of phenomena connected with movement. The existence of certain invariant of "events interval" are exhibited in most of narrations of ST as "undeniable proof" necessity to application the "space-time" (as a kind of unobservable reality in fact):

$$
s^{2}=\Delta r^{2}-c^{2} \Delta t^{2}=\left(\Delta r^{I}\right)^{2}-c^{2}\left(\Delta t^{I}\right)^{2}
$$

where: $s$ called "events interval" (or, space-time interval); $\Delta r, \Delta t$ and $\Delta r^{I}, \Delta t^{I}$ are differences of spatial and time coordinates between events in two frames accordingly, moving relative each to other. The invariance principle is written as below in Cartesian coordinate frame:

$$
\Delta x^{2}+\Delta y^{2}+\Delta z^{2}-c^{2} \Delta t^{2}=\left(\Delta x^{I}\right)^{2}+\left(\Delta y^{I}\right)^{2}+\left(\Delta z^{I}\right)^{2}-c^{2}\left(\Delta t^{I}\right)^{2}
$$

where: $\Delta t, \Delta t^{I}$ and $\Delta x, \Delta y, \Delta z, \Delta x^{I}, \Delta y^{I}, \Delta z^{I}$ are time and space coordinates differences in two relatively moving frames. Assuming $\Delta y=\Delta z=\Delta y^{I}=\Delta z^{I}=0$ we write:

$$
\Delta x^{2}-c^{2} \Delta t^{2}=\left(\Delta x^{I}\right)^{2}-c^{2}\left(\Delta t^{I}\right)^{2}
$$

Applying $\left(\Delta t^{I}\right)^{2}=\Delta t^{2}\left(1-V^{2} / c^{2}\right),\left(\Delta x^{I}\right)^{2}=\Delta x^{2}\left(1-V^{2} / c^{2}\right)$ as per LT, from (2.19) we get:

$$
\Delta t=\Delta x / c
$$

(2.20) shows: the light velocity and distance are actual factors that causing difference between results ST and classic laws to the phenomena connected with movement.

Meantime, we saw that the same factors brought to observed distortions of reality in consequence of the movement (2.1.2). Equal results of these factors and interpretations show that the dealt relates to the same phenomena. We need remark that similar explanation to creation of LT and ST as result of misinterpretation of averaging the light velocity on directions "ahead and back" contains in Canadian astrophysicist Paul Marmet' paper also in Ref. [12], next with mentioned remark Ref. [3]. The reason and necessity to removing unnecessaryunclear concepts from our lexicon becomes obvious with the same.

\section{5) The "space-time" and non-Euclidean geometry}

Other mystic attractive concepts and terms have appeared in different divisions of natural science with creation of SR. We would briefly examine the gist of an important one of these by using previous judgments and conclusions. One of the most known affirmations of SR concerns to the oldest science of geometry. It has been declared in SR somewhat different from "Euclidean" that was long believed as doubtless. Reader can easily comprehend the essence of the question from the previous content. The matter is Euclidean geometry is built on certain axiomatic basis where the static system or, our possibility of instant measurements is supposed unspoken. The issue is that possibility of instant measurement is silently accepted in classical physics as an idealized priory supposition. We can comprehend that it does not change anything in our observations in the static world, i.e. if studied objects do not move in relation to the observer (or, the relative movement insignificantly "slow" compared to the velocity of light). Thus, Newton's mechanics as well as Euclidean geometry are based on the mentioned silent convictions. It becomes comprehensible that their basic principles will seem as "somewhat distorted" in dynamic world, i.e., when the measured objects move with relative speeds compared to the velocity of light. It is easy to comprehend that the observable deviations will depend on two factors. These will be the in- 
crease with the speed of the movement and decrease with the information transfer speed; thus, the relative difference will look as some function $A^{I} / A=f(V / \mathrm{c})$, where $V$ is the speed of object; $c$ is the light velocity. The above examined though experiments have clarified some aspects of "distortions" of real values pointing on the essence and circumstances of creation of SR. We can comprehend that inevitable deviations of observable results from Euclidean geometry will arise with the movement of studied objects (i.e. in dynamical system) because of the distortion of measured distances and objects' locations relative to actual ones (2.1.2). We already know the causal character of the difference between observable and actual pictures of reality (1.1. f)). Therefore, we can comprehend that it is not necessary to declare Euclidean geometry as a "conceptually wrong" science that needs to be replaced by some other kind. Meanwhile, we must just consider the limited speed of measurements (observations) that causes the mentioned differences between the observable and actual pictures of subject phenomena. Then we get new description rules and geometric laws where the inevitable errors of observations are considered. We can call those as "pseudo Euclidean" or "Lorentz geometry" if desired, comprehending however, that it is actually the same Euclidean geometry, where the errors of observations are considered. The "new geometry" gives us opportunity to make the calculations and get results that coincide with observations. However, we lose another important capability with the same. The matter is, we cannot use the description by "new geometry" for the cause-effect (or logical) investigations of phenomena because it contains the errors of observations

What do we need to do in such situation? Copernicus already gave the exhaustive answer in time that may be applied to this case also (1.1. f)). We must just clearly divide the values, descriptions and pictures into observable and actual ones. Then we can understand where and how to use each of these correctly as well as how to pass from one kind of values to the others. Namely, if we need to investigate the cause-effect side of phenomenon we must recover the actual picture from its observed one, by taking into account the errors of observations. These arise because of limited velocity of light, in context of the studied problems. We can recover the picture of the phenomenon that we can see if the velocity of light will be infinite. The picture that we get in such a way will correspond to its description in the idealized Euclidean geometry that can serve us in the cause-effect investigation of phenomenon. However, we need to "go back" again to "Lorentz geometry", where limited velocity of light is considered, to get the opportunity to compare our conclusions to experimentally-observables. Thus, the task and operations of such transformations from one kind of geometry into the other are principally similar to that used to study the movement of planets, using the geocentric-heliocentric-geocentric systems transitions, the significance of which is clear to us and does not call for any questions. We just need to realize the flaws of our observation system in one case, and the imperfection of our measuring tool in other, which we must consider in our actions and judgments to get correct conclusions. The matter is we have put the questions in different ways in formal and in realistic methodology. In the first case, we wish to have the description of the phenomena, which coincides with our observations, as it seems to us, without thinking of its cause-effect side. However, in the second case, we wish to penetrate into cause-effect essence of the studied phenomenon. Then we must do some additional operations with our results of observations to "filter" them from inevitable errors connected to imperfections of real systems and measurements. The imaginary ("clean") picture of the phenomenon in the idealized system of observation only can to serve us for its causal investigation and correct conclusions.

6) On the significance of $\mathrm{SR}$

We have shown above that the cognitive problems of SR were arisen due of trivial confusions of used concepts as well as with the arbitrary interpretations. We hope this explanation may be easily perceivable, despite the whole painfulness and the huge psychological problems related to suggested recognition.

i) We see the most negative role of $\mathrm{SR}$ in introduction of the uncertain concept of "space-time" that has prevented the development of subject divisions in physics

Meanwhile, we see inexcusable to announce SR as some "reactionary and totally harmful falsification that needs to be excluded from natural science at all" as demanded by most critics and hard opponents of Einstein's theory. We can emphasize next, with all criticism and marked flaws, some of the new visions and non-traditional innovations of SR that give undeniable push to resolving certain huge problems as:

j) SR provides quantitative descriptions of certain phenomena that mostly coincide with observable results; it may be used at applied-engineering level. $E=m c^{2}$ fundamental relation is one of the undeniable huge shifts in natural science

We also remark next unprecedented innovation of SR from methodological viewpoint:

k) The description of phenomena in different frames of observation with comparison of their results gave principally new opportunity to reveling unknown relations of nature 
The opening of mass-energy communication was possible to reveal thanks to studying the same phenomenon in two systems of observations, as for example. Thus:

We need to perceive SR within its actual significance: as a way of description of phenomena where our real capabilities and admitted inevitable errors of measurements-observations are considered, next to cognitively irrelevant interpretations that need to be replaced by cause-realistic ones.

\section{Physical Essence of Gravity}

I believe The Lord has decided what we need to understand and what not, but allowed us to try!

Author

\subsection{Acquaintance to Stated Problem}

We ubiquitously see free falling of things and feel their weight, perceiving these quite ordinary, not having any idea and easily neglecting the causal essence of the oldest mystery of nature, called "gravitation". Such preamble may sound outrageous and unexpected for many, on the background of frequently launched rocket-satellites at present with applied or research purposes. We also periodically have learnt about next confirmations of this or that predictions of Einstein's famous theories and of other remarkable achievements, closely related to examined subject demand a serious theoretical base and sophisticated calculations, inspiring the opposite impressions. Meanwhile, the complete darkness of physical gist of gravity is today's reality in natural science and our reader must take this allegation seriously, resolution of which is one of the main tasks of suggested work. The stated problem somewhat dropped out of attention at present, in comparison to researchers' early attempts devoted to open the physical essence of the gravity. Present efforts and huge means however, are mostly directed to study of the quantitative side of the gravity phenomena in fact, due to certain historical circumstances, as noted (1.1.):

a) We strive to define the causal basis of the examined phenomenon initially, from which its quantitative peculiarities can be derived naturally

The Great Newton actually did not tell anything on the physical nature of gravity in his time, proving just that we get the right description of the observable movement of material bodies and the known celestial mechanics supposing far-acting forces in Inverse Square of their distance (1.1). Newton's gravity does not give answers to natural questions: what is the nature of that force? Through what environment and how it passes there? Meantime, the mystical force, instantly acting on unlimited distance, looks unnatural and it was perceived by theorists skeptically from the beginning. The concept of "gravity field" was introduced in physics with Newton's gravity, as a "transmitter" of the hypothetical far-acting forces in fact. It has been presented uncritically within close analogy to Coulomb's electrostatic field in most textbooks, using similar terminology (as the "gravitational potential"). The "gravity field" has been looked as a kind of physical reality, as "it having its own peculiarities", that has only hindered the actual cognitive problem for a long time.

We can look at some of the new theories with involvement of Mach's Principle as contemporary modifications of far-action and field-based theories of gravity in which the instant action has changed with finite speed of gravity influence propagation equal to light velocity as Ref. [13]. Mach's Principle however, must not be acceptable for us since it also obviously supposes the existence of a new kind of physical reality (as influence transmitter) without any experimental evidences of its reality (see point c) below). We need to emphasize however, the mentioned approach gives quantitative results equivalent to Einstein's GR. By the same, it may serve to us as an additional testimony of the causal essence of gravity in our further attempts.

b) Le Sage's theory of gravity was one of the conceptually formulated explanations of the essence of gravity, from the contemporaries of Newton. It was based on the existence of kind of special hypothetical particles that move in all possible directions in space with much more speed than light velocity, arousing the screened (or shielded) effects between material bodies, being partially absorbed in these. Le Sage's particles however, did not stick because of different serious counter arguments, one of that pointed by Poincare Ref. [14].

c) We will briefly examine here the contemporary modification of "special particles" theory of gravitation which is related to introduction of mass-less gravitons as the mediators of force transmission at distance in quantum field theories. Many modern works have been developed with the gravitons now. Meanwhile, direct detection of single gravitons experimentally seems practically an irresolvable task because their energetic insufficiency. An indirect confirmation of their existence by detection of gravity waves (as their coherent groups) seems realizable; practical works on this direction are underway at present (see LIGO, VIRGO, LISA experi- 
ments etc.) We will not examine the technical base and disputes on this matter referring to existing large literature on the subject as example Refs. [15] [16]. However, we shall pay serious attention to the fact that experimenters have been looking for gravity waves for significantly long time. The used techniques are much improved (with the costs) starting with earth-based antenna-detectors and passing to cosmic interferometers having incomparable capabilities at present. The gravity waves however, remain undetected yet, despite the achieved fantastic sensitivities of detectors. One alarming factor here is that the different significances of gravity signals are accepted within time. The suspicious conclusion simply follows then without touching the technical details of the problem at all. The theorists are doing their calculations issuing from certain assumptions that give certain initial data. The experiments, however, do not confirm their predictions in fact. Then, they change the initial data and their basic supposition too, to get other result than the earlier adopted one (in this case much smaller energy of gravity waves). It shows that apparently, theorists are not even working by test-error principle (1.2), and they just strive to adjust their calculations to the results of experiments i.e. without any definitive base concept. Mentioned circumstances do not correspond to initial criterions of objectivity and methodology of realistic science that have been declared. Therefore, we can appreciate them as unreasonable expenditure of efforts and means. It seems appropriate to recall here the practice of introduction of hypothetical kinds of realities to explain the incomprehensible phenomena, largely used by early thinkers in complicated cases; these mostly brought nothing but irresolvable mysteries. The known histories with the "phlogiston" and with kinds of "ethers" may serve as examples.

The Great Newton said: "Hypotheses non fingo" (I contrived no hypotheses). We can interpret this famous expression as a transparent commandment-do not harm natural science by own compositions! It seems as pertinent to refer to the similar opinion on harmfulness of "unnecessary essences" (see Occam's razor) as in Ref. [17]. Mentioned principles express the reasonability and mandatory the preference in natural science of confirmed facts relative to arbitrary suppositions, by authors' interpretation. Thus, above-examined critical remarks and accepted methodological criterions gives to us full rights to reconcile with lack of gravity waves and to think; what would that mean?

d) The absence of gravitational waves exacerbates problems with gravity much more, at the first glance. From other side however, it may give to us next valuable instruction:

We search the causal explanation of gravity problem on completely wrong direction since recognition of absence gravity waves demands basic changes of the considered versions and representations on the physical essence of gravity in whole.

The existence of "gravitons" and "gravitational waves" accepted to represent as the derivatives from GR. Meantime, the famous theory of Einstein-GR remains yet without any of unambiguous causal explanation as noted; it gives large possibility to interpret this or that its quantitative results in arbitrary manner, by theorists' personal propensity (we see it actually with presence several different theories in similar quantitative significance!).

The tremendous cognitive revolution has been predicted by few authoritative specialists on this area, needed to open physical essence of gravity phenomena as Ref. [18].

The GR (and equal theories) are adopted by specialists as the adequate quantitative description of gravity phenomena at present, most results of which are confirmed experimentally, excluding the "gravity wave" that we will discuss.

Authors share the majority's opinion; Einstein's theory gives sufficiently correct quantitative descriptions to many observable effects, related to gravity phenomena. We emphasize however, that GR remains as completely non-trivial from the causal-cognitive viewpoint, the illumination of which is one of the main tasks in the work.

e) The statement of question is possible to present as the following allegation:

GR (and equal theories) are satisfactorily correct theories in quantitative meaning. Therefore, these must have enough informative content to open the causal essence of examined phenomena as well, in virtue of their correct quantitative relations.

Above-said is right to assert in relation to Newton's gravity as well, with consideration of it as the approximation of GR. The mentioned opportunity and advantage follow from the adopted methodology, with the demand of parallel usage of mathematics and logical considerations in research process (1.2. a)). It allows translation and passage from one kind of language and description to another, upon necessity. Thus, we just need to define the correct physical meaning of used mathematical symbols and actions, to pass to the descriptive language and causal interpretation of gravitation from the correct quantitative relations that are already known to us. 


\subsection{The Causal Side of Gravity}

We shall briefly examine some interpretations and terminology adopted in GR and other gravity theories from cognitive viewpoint that may hint on the essence of the problem. We shall remark first that Newton's "instantly far-acting" force was removed in GR that we see as an important advance in cognitive meaning: as a rejection of arbitrary hypothesis!

a) The gravity phenomena are interpreted in GR as non-trivial consequence of curvature of the "spacetime", as presented in most literature. It creates the following impression and causal picture of the gravity problem, if guided by pure formal logics:

A special kind of reality, "space-time" exists that becomes "curved" in surroundings of a central massive body under its influence. The "curvature" of "space-time" acts on test material bodies forcing them move to central body by acceleration (free falling), or be pressed on its surface (weight force) after their collision.

Meantime, we can just state the absence of any experimental results for today that directly evidence the existence of kinds of physical realities corresponding to demanded peculiarities of "space-time".

It is easy to comprehend also that the same facts could serve for proving the reality of Lorentz' ether, Le Sage's particles, as well as the existence of gravitons etc, if these could have been observed in its time. The next formal interpretation also adopted that sounds as:

The pseudo Euclidean Lorentz geometry (2.1.5) turns into Riemannian under the influence of material substance. Spatial geodesic lines are peculiar to it and material bodies move along these free from force influence, in the form of orbital movement.

Then some impressions and corresponding interpretation arise, such as "the Riemannian geometry causes gravity phenomena at all"! We hope it will be easy for the reader to comprehend the absence of any physical meaning in this formulation from previous content. We just need to state here that "geometry" is a kind of science, to comprehend above-said. Then it becomes clear that "geometry" (as well as any science) is a way of description (tool, language, system, i.e. a human's abstract creation) that itself is unable "to influence" the physical reality! We can see here a simple confusion of abstract math and real physical concepts [3], creating a nonsense, peculiar to formal methodology $(1.1,2.1)$. Thus, we can state the actual absence of any third real physical participant in the gravity phenomena, being observed between two objects, independent from interpretations and used terms (as "special particles", "physical fields", "ether", non-detectable "space-time", "curved geometry" etc.) We see that non-ordinary terminology and concepts of GR are conditional names only to mark some math objects and actions. Thus, it will be meaningless to use these for causal description, because of the absence of their initial physical meaning at all.

b) The actual significance of "space-time" in SR is reduced to consideration of light velocity factor in our measurements (observations) in a form of universal correcting coefficient $\gamma(2.16)$ as it is shown in the previous chapter. We show above, the movement of studied objects and limited light velocity has caused the difference between Newton's physics and SR. We can get convinced that all of the confirmed gravity effects of GR, distinguishing it from Newton's gravity strive to zero if we accept $c \rightarrow \infty$, which means GR turns into Newton's gravity, same as SR turns into Newton's physics. Above-said, however, transparently instructs that GR effects are the consequences of a certain dynamic process, from the logical viewpoint. It just means that gravity effects are conditioned by the movement (as SR effects were). The argumentation of this conclusion is obvious; if we deal with static world and unmoving objects, we will get the same results of measurements independent from speed of our measurements, i.e., the light velocity should not be expressed in experimental results and in our formulas! (It means our geometry always will seem Euclidian). We shall compare some known expressions of SR and GR to show the rightness of this conclusion. The invariance of elementary interval of "space-time" is written in SR as:

$$
\mathrm{d} s^{2}=(\mathrm{d} x)^{2}+(\mathrm{d} y)^{2}+(\mathrm{d} z)^{2}-c^{2}(\mathrm{~d} t)^{2}=\left(\mathrm{d} x^{I}\right)^{2}+\left(\mathrm{d} y^{I}\right)^{2}+\left(\mathrm{d} z^{I}\right)^{2}-c^{2}\left(\mathrm{~d} t^{I}\right)^{2}
$$

where, the relations for elementary spatial and time intervals in two frames are the same:

$$
\frac{\left(\mathrm{d} x^{I}\right)^{2}}{(\mathrm{~d} x)^{2}}=\frac{\left(\mathrm{d} y^{I}\right)^{2}}{(\mathrm{~d} y)^{2}}=\frac{\left(\mathrm{d} z^{I}\right)^{2}}{(\mathrm{~d} z)^{2}}=\frac{\left(\mathrm{d} t^{I}\right)^{2}}{(\mathrm{~d} t)^{2}}=\gamma^{2}=1-\frac{v^{2}}{c^{2}}
$$

We mean $v$ in (3.2) as the free variable by its definition and all of SR effects were simple consequences of 
movement as shown in previous chapter. It is easy to observe that GR consequences and effects may be represented as similar functions of $v / c$ relation. The linear element for spherical-symmetric Schwarzschild metric, for example, presented as:

$$
\mathrm{d} s_{s}^{2}=c^{2} \mathrm{~d} \tau^{2}=\left(1-\frac{r_{s}}{r}\right) c^{2} \mathrm{~d} t^{2}-\left(1-\frac{r_{s}}{r}\right)^{-1} \mathrm{~d} r^{2}-r^{2}\left(\mathrm{~d} \vartheta^{2}+\sin ^{2} \vartheta \mathrm{d} \varphi^{2}\right)
$$

where: $1-r_{s} / r \approx e^{v}$ characterized the "space-time curvature", $r$ is the distance of point from centre of material body $M$, and $G$ is the gravity constant. The $r_{s}=2 G M / c^{2}$ called the Schwarzschild radius by accepted terminology in GR. We can write:

$$
e^{v}=1-\frac{r_{s}}{r}=1-\frac{2 G M}{c^{2} r}=1-\frac{v_{g}^{2}}{c^{2}}
$$

The physical meaning and significance of speed $v_{g}$ will be examined next. We shall mark only that it is not free variable in GR as it is in SR, and $v_{g}$ defined by parameters of material substance as (3.4). Thus, the identical structures and values of $e^{v}$ and $\gamma^{2}$ factors evidenced:

c) GR effects and the term "curvature of space-time" (or "gravity field" etc.) must be comprehended in the same meaning as the effects of $\mathrm{SR}$; i.e. these are observable distortions of reality caused by the objects' movement and by limited light velocity

Thus, this conclusion is justified from both sides: from ordinary logical viewpoint as well as from purely formal consideration, in virtue of the same physical values and their same combinations cannot be interpreted in different ways, in any scientific methodology. The possibility of similar transformation (3.2) and (3.4) as function of $\mathrm{v} / \mathrm{c}$ can be observed in all confirmed effects of GR, without any exception, as for the displacement of planets' orbits, gravitational change of frequency, frame dragging effects etc., that also confirm the above conclusion (examples are shown next). Thus, we come to a clearly formulated next conclusion:

-The gravity phenomena are consequences of an unknown to us movement.

The known Einstein's Equation (3.5) in GR are perceived and interpreted by theorists as the "field's equations".

$$
G_{\mu v}+\Lambda g_{\mu v}=\frac{8 \pi G}{c^{4}} T_{\mu v}=R_{\mu v}-\frac{1}{2} g_{\mu \nu} R+g_{\mu v} \Lambda=\frac{8 \pi G}{c^{4}} T_{\mu \nu}
$$

The structure and the physical units ${ }^{7}$ of components in (3.5) simply show, however, GR equations are related to a motion and nor to kind of physical reality as it declared.

Then the "gravity field", "curved space-time", different "special particles" etc. become groundless hypotheses and arbitrary interpretations in fact. This conclusion however, immediately collides into incredible problems. The matter is we do not see any kind of movement that may directly confirm it. We observe, for example, weight of things in their obvious unmovable condition and we cannot imagine on what kind of movement we can talk here. We shall however put aside different "obvious" questions to continue ubiquitously examining our conclusion unequivocally deriving from examined arguments.

d) The "local equivalence of gravity with inertia" (Einstein's equivalence principle) (EEP) is adopted as the other most important basic principle in creation of GR

We shall firstly mark the mysterious character of the adopted allegation from logical viewpoint, that we have the right to discuss, because the used terms "gravitation" and "inertia" are exclusively concrete physical concepts demanding clear definitions of their meaning. Ordinary reasoning tells us that real physical objects can be individually independent things or, these may be the same thing with different names only. It will be an obvious nonsense to say something as; "the objects $A$ and $B$ are individual at all but may be the same things within some conditions!" some numbers as equal to actual, or by adopting the curve element as "straight" in differential calculus. The matter is such approximations have meaning if compared objects are of the same kind, otherwise we will fall into obvious nonsense (as if comparing "mass" and "distance"). This demand is preserved in above examples, as

\footnotetext{
${ }^{7}$ A pure mathematical, unitless form of quantitative expressions is used in formal methodology that opens way to interpret the physical meaning of results in own vision [1].

${ }^{8}$ Academician A. A. Logunov wrote about equivalence principle, "I am not able to understand it"!
} 
compared concepts are both of the same kind ("numbers" or, "lines"). The concept of "inertia" we can define as a phenomenon only, arising in consequence of accelerated movement, thus as a dynamic process. Then we can conclude:

-We are obliged to consider the phenomenon of "gravity" as consequences of accelerated movement, guided by the demand of uniformity of compared concepts.

Thus, we got one more independent instruction of the accelerated character of unknown movement (3.2. b), c)) that causes the "gravity" phenomena.

We shall present now the circumstances that have pushed for the acceptance of the mentioned strange allegation in GR. The phenomenon, caused by accelerated movement, well known to us from Newton's mechanics. The most important certain peculiarity of the inertial mass of material objects is revealed in the phenomenon, characterizing how the body resists to external force and acceleration. It may be experimentally defined from Newton's second law $F=m_{i} a$ as:

$$
m_{i}=F / a
$$

where: $F$ is the acting external force; $a=\mathrm{d} v / \mathrm{d} t$ is the acceleration of movement.

The number of experiments, starting from Galilee and further much more exact ones, has shown that gravity forces acting on the material bodies are defined by another common peculiarity, independent from the kinds of tested materials. The gravity force that acts on the test body is defined by Newton's law of universal gravity as below:

$$
F_{g}=\frac{G M m_{g}}{r^{2}}=k m_{g}
$$

The $m_{g}$ in (3.7) is called the gravitational mass that characterizes corresponding peculiarity of test body; namely, it shows how much weight force the body generates under the gravitational influence, the physical nature of which is still unknown to us. Thus, the above-mentioned experiments show unequivocally that these two different experiments give exact equal values for the mentioned two kinds of characteristics of the substance:

$$
m_{i}=m_{g}=m
$$

The equivalence (3.8) brightly expresses all the mystery of gravity phenomena since the natural question arises there why these two kinds of characteristics must be the same that remains unanswered, despite the big number of theories, uncountable written pages and long disputes. Moreover, the reader must know that many other kinds of experiments with the gravity and with the accelerated movement give the same results:

-The existing large group of facts shows that the consequences of "gravity" influence are indistinguishable from those that arise in consequence of accelerated movement.

It concerns the above-mentioned phenomena: 1) force influence and movement (i.e. weight and free falling); as well as 2) geometrical changes of light's trajectory; 3) the gravitational change of light frequency; 4) the gravitational delay of time and other effects. Mentioned equivalence of the gravity and inertia are mainly confirmed by number of experiments that reader can find in the literature, as for example Ref. [19]. The possibility of representation of the known effects of GR as the consequence of accelerated movement is also shown. Einstein has taken into attention the mentioned experimentally established fact in creation of his gravity theory as a key principle that has been formulated in EEP. We invite reader's attention to the next important remark once more. The matter is:

-Einstein did not explain any way the similarity of the two kinds of phenomena (gravity \& inertia) that were adopted as different subjects with initial definitions; and he only stated the experimentally revealed facts, considering these in the base of GR.

Moreover, the genius thinker never hides that he does not comprehend the causal essence of gravity phenomena; he says, for example, "If I could only understand what goes on in a falling lift!" Ref. [8]. Thus, from previous content, we shall evaluate Einstein's relativity theories as they actually are, i.e., as pure formal-mathematical ways that provide important results, mostly corresponding to observations. Therefore, the attempts to present our conclusions as somewhat opposing Einstein's relativity theories will be obviously inappropriate and groundless, because of actual absence of any causal interpretations there at all, by definition (1.1 b)).

e) We shall examine now the important question: where from arises the restriction of "locality" in EEP? Named restriction only hindered direct identification of two concepts (inertia and gravity), which may signifi- 
cantly change created situation. The logical vague character of EEP has pushed some theorists to reject it at all, accepting inertia and gravity as separate phenomena having nothing common with each other. In fact, the gravity phenomena are attributed completely to peculiarities of "space-time" in RTG as Ref. [20] ignoring mentioned principle for example. Meanwhile, the attempts on opposite direction have taken place in other works, by presenting "inertia" as a consequence of "curved space-time" also, to explain the similarity of the two phenomena. The presentation of "space-time" as "owner of peculiarities" however, simply transforms it to a kind of hypothetical physical reality, on the rank of "non-provable ethers", the wrongness of which was shown in previous chapter. Moreover, by this approach, a large group of equal results of mentioned experiments (3.2. d)) seems as a number of unexplainable exact coincidences. Thus, from above argumentations we see the inevitable necessity of a clear definition:

Is the deep similarity of gravity and inertia phenomena a pure coincidence or are they identical by their physical essence with different names?

The first choice seems just unbelievable with elementary logic, by taking into consideration the equivalence of inertial and gravity masses only, leaving aside even the large group of other kinds of coincidences. The conclusions on equivalence of gravity and inertial masses of Galilee and Newton had been confirmed by Eötvös with impressing accuracy $\left(10^{-8}\right)$ about hundred years ago, later with much more exactness $\left(10^{-11}\right)$ Ref. [21], and known last results were achieved in $1999\left(10^{-14}\right)$ Ref. [22]. However, new projects to test the equality of gravitational and inertial masses (weak equality) with unprecedented accuracy $\left(10^{-18}\right)$ are suggested at present as Ref [23]. Meanwhile, the above-described reality clearly shows that the ubiquitous similarity of gravity and inertia phenomena are accepted by researchers as a statement, as they examined the question "how similar they are", and not "why they are similar". Thus, from cognitive viewpoint we can state:

The restriction of "locality" in the equivalence principle has banned direct identification of the concepts of "gravity" with "inertia", which caused further huge cognitive complications.

However, scrupulous examination shows actual absence of any quantitative exposition of mentioned restriction of "locality" in GR. Reader himself can get convinced that nothing changes in GR if we replace the "local equivalence" by direct identification of "gravity" with "inertia". We see in GR $\mathrm{m}_{\mathrm{i}}=\mathrm{m}_{\mathrm{g}}$ (3.7) adopted without any conditions or criterions; it shows that the concepts of "gravity" and "inertia" are actually indistinguishable in quantitative meaning. Thus, pure verbal-psychological character of the "locality" restriction becomes obvious from above-said. The mentioned fact is obvious and can be checked up if desired. Some critics of GR have also observed that concepts of gravity and inertia are quantitatively indistinguishable as Refs. [20], [24]. Moreover, academician Fock had pointed in his book on EEP; "The law of equivalence of inertial and weight masses have a general and nor a local character" in Ref. [25]. We need to emphasize that a number of experiments confirm the exact equality of gravity and inertia but not the "locality". The fictional essence of the restriction of "locality" gets obvious; we can assert:

Experimental results as well as quantitative expressions, confirming restriction of "locality" in equivalence of gravity with inertia are absent in GR. Thus, the allegation of "locality" may be evaluated as a verbal declaration, adopted in virtue of intuition.

We briefly examine beliefs and prejudices induced to adopt the restriction of locality in EEP that hindered the acceptance of gravity and inertia as the same thing when facts and reasons to do it are many. We know that the results of experiments in the accelerated spacecraft and in the unmovable lab in gravity field are the same; by free falling of test bodies, by force reaction (weight), by deflection of light path etc. Mentioned similarity of results does not allow the inner observer to define whether his closed lab is in accelerated movement condition or it is in corresponding gravity field? The equivalence principle was adopted due to similar results. However, the solution of the problem and detection of the difference between gravity field and accelerated movement seems to be possible if we use an "enough big" lab (or enough exact measuring tools). The trajectories of falling bodies for example, directed to the centre of material body, being the source of gravity field that are not parallel (Figure 3(a)). Meanwhile, these are supposed to be parallel in the accelerated spacecraft (Figure 3(b)).

The above-described conclusion seems sufficient to put the restriction of locality in equivalence to gravity with the accelerated movement. This conviction however, is completely based on supposition because the difference between gravitational and inertial phenomena is not yet confirmed experimentally anyway. This restriction also does not have any quantitative expression in GR in fact, as noticed above. Then we mark:

The restriction of "locality" in GR plays purely declarative-psychological role, introduced because we do not see directly the corresponding movement. 


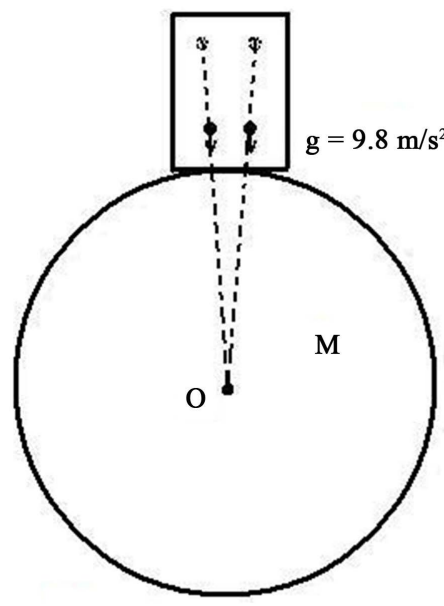

(a)

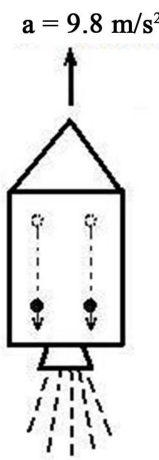

(b)

Figure 3. The difference of trajectories in free falling (a), and in the acceleration (b).

\section{f) The identity of the concepts of gravity and inertia: universal expansion of substance}

The concepts of "gravity" and "inertia" are actually used in the same quantitative significance in GR, examined above. This conclusion opens clear indication of the physical nature of gravity. Moreover, it is easy to see that the problem of "locality" by itself goes out of agenda if we adopt the "gravity" as the "inertia", sacrificing our intuition. Thus, the whole group of phenomena inside our terrestrial lab that we call "gravitational", can be looked as consequence of accelerated movement. It follows that our Earth, for example, continuously expands pushing the things on its surface in radial directions with acceleration, and the bodies resist to acceleration with inertial forces according to second law of Newton. The free falling of different kinds of test bodies with identical acceleration becomes simply explainable; the things, being free from their supports actually remain in their former places; the surface of Earth reaches them simultaneously.

However, we will be unable to detect the described expansion visually due to its universal character for the material substance at all. The cabin of accelerated spacecraft as well as our etalon meter will expand proportionally with all other material objects for the same reason. The trajectories of free falling test bodies will be indistinguishable from those, which have been in the terrestrial lab as illustrated in the graphic, i.e. they become not parallel (Figure 4). Such explanation of gist of gravity strongly contradicts to human intuition due to our daily perception of material world that directly hinders even its detailed study.

Many "obvious" objections also immediately arise there, for example such as: how can orbital movement and the celestial mechanics be explained, replacing universal attraction by expansion of material substance? The problem, however, is not new in the history of science from cognitive viewpoint. The humanity, for example, has been forced to agree with the "rotating" Earth and its orbital movement, with incredible velocity that was "the most unmovable thing" for us. We cannot see this, and we have adopted it today as out of doubt-after paying a proper price! Then it is possible to comprehend that we are in the same situation; we need to pay the next huge price, going versus our natural intuition to solve the mystery of gravity. Different kinds of gravitational phenomena then become possible to interpret on the comprehensible causal base, without exceptions, (some examples follow).

The concept of proportionally expanding material universe gives us important evidences of solution of many other fundamental problems of physics that will also be discussed further. Firstly, however, we shall draw the attention to below historical comparison: we have intuitively formed many "doubtless" convictions on surrounding us material world due to our direct perceptions. We were initially convinced in the:

1) Absoluteness of rest and movement; 2) absoluteness of directions; 3) opportunity of absolute (instantly) observation-measurement; 4) absolute invariable sizes.

We learnt the history and dramatic events that forced us to remove the first two points from our minds from school education. We tried to show in the previous chapter that logical problems with SR reduce the necessity of releasing the third point from this list of false convictions. The fourth point however, remains yet strongly unshakable in our mind that forces us to refer to inappropriate creations to save our conviction, arisen because of natural intuition. 


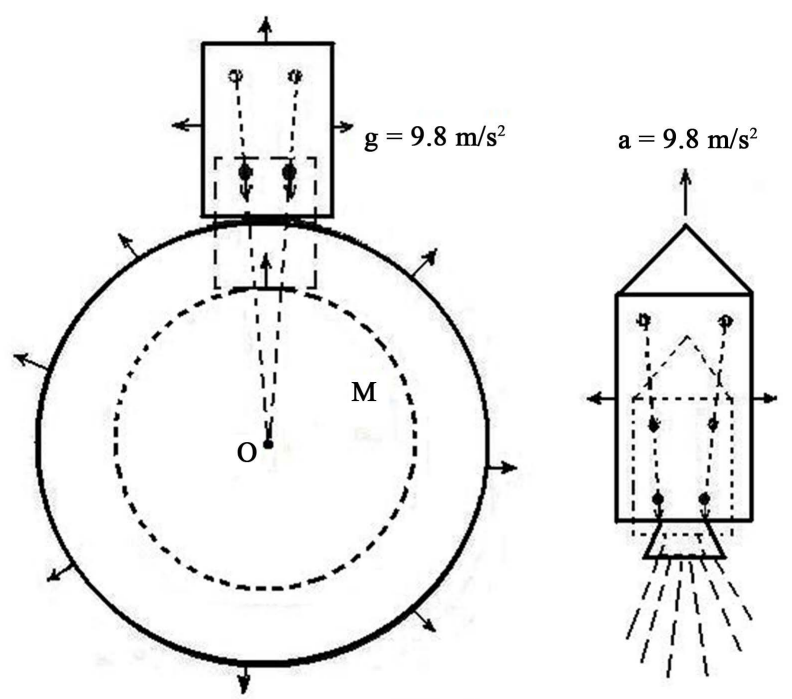

Figure 4. The full equality of expansion with the grawity.

\section{g) Other evidences on expanding world}

1) The causal interpretation of physical nature of gravity is absent in Newton's gravity theory as mentioned above (3.1. a)). Meantime, the actual identification of inertial and gravitational phenomena is silently used there in fact, since Great Newton does not put any difference between the two kinds of masses in his theory and quantitative considerations at all. It simply brings to the same conclusion in favor of expanding world, as it was narrated above.

2) We can find the valuable evidence of universal expansion in Ref. [13]. This theory provides correct results equal to GR (3.1 a)) and the conceptual explanation of gravity is based there on the variability of particles' mass. The brief content of theory is as below:

All kinds of physical values are possible to represent by combinations of $h, c$ natural constants and with a single basic value only, serving as free parameter, having measures L, T, M, etc. This possibility derives from well-known quantum relations:

$$
m=\frac{h}{c \lambda_{c}}=\frac{h v_{c}}{c^{2}}=\frac{h}{c^{2} \tau_{c}}
$$

where, $m$ mass of particle; $h$ Planck's constant; $\lambda_{c}, v_{c}, \tau_{c}$ are Compton's wavelength, the frequency and the wave period, accordingly.

We need to emphasize here that the free parameter may be variable, in virtue of its uniqueness. The laws of nature and observed phenomena in general will remain the same with this supposition (it means we will be unable to see any changes in our world). This conclusion was used in mentioned work by accepting the mass as a variable. However, the relations (3.9) unequivocally say that $\lambda_{c}\left(v_{c}, \tau_{\mathrm{c})}\right.$ also must change with variable mass of the particle! Thus, we are just obligated to adopt continuous expansion of Compton's wavelength and decrease of its frequency, if we accept continuous reduction of the masses of particles. It means our world is in dynamically variable condition, i.e. our meters and our clocks etc are permanently in change together with us and with all of material things, in such a way that leaves no opportunity for us to perceive our real situation directly. We observe reaction of forces between contacting material bodies (weights) and we see their "free falling" each to other that remains completely unexplainable to us due to our intuitive convictions! The difference of two theories (GR and [13]) relates to their verbal interpretations only having no actual significance in the results; thus, with the same, the described conclusion on expanding world relates to GR as well.

3) Hubble's expansion of universe (Hubble's flow), that is now accepted as a doubtless fact by dominant majority of experts, may serve as direct evidence to expanding world.

It was established by observations that far away galaxies recede from us with some speed proportional to their distance. This is characterized by Hubble's law; $\mathrm{V}_{\mathrm{H}} \approx \mathrm{H}_{0} \mathrm{D}$, where $\mathrm{H}_{0} \approx 75(\mathrm{~km} / \mathrm{s}) / \mathrm{Mps}\left(\mathrm{Mps} \approx 3.09 * 10^{22} \mathrm{M}\right)$, and $D$ is the distance to observed object. We need to bring only one important remark on this matter. The dealt is 
a strange situation created by this wonderful opening, arousing continued disputes among theorists. The expansion of universe actually follows from GR (it is simple to understand from previous content). It was theoretically shown particularly by de Sitter, A. Friedman, G. Lemaitre. Einstein added then the special constant $\Lambda$ (cosmological constant) in his Equations (3.5) for the sake of "protecting the static condition of universe". However, its necessity disappeared with the opening of Hubble's expansion. Thus, the following picture has been created:

The "gravity influence" pushes universe into compression, vs. expansion of Hubble. The intriguing question immediately begs here: which of these opposing factors will prevail in the fate of universe? Then the experimenters have begun the measurements of density of substance in universe for necessary correction of Hubble's constant to solve the arisen problem. The statement of the question is the following: is it the less or exceeding average density of substance than the critical ratio in universe that will define how the expansion will go in future? Will it continue forever or it will stop and change to compression etc. The surprising fact however, has revealed; the corrections show that relation $f\left(H_{0}, \rho\right) \rightarrow f\left(H_{0}, \rho_{c r}\right)$, which means the factor of expansion has appeared too close to gravity compression factor that does not allow yet to answer clearly what will happen to universe in future. The solution of problem is possible by analyzing the above-mentioned facts.

The universal expansion follows from GR i.e. from quantitative description of gravity. It just means that both of these factors are different expressions of the same phenomena; their equal quantitative significance becomes then simply explainable.

Moreover, the accelerated character of expansion of universe also has been confirmed by observations as in Refs. [26] [27]. Thus, we have a complete opportunity to replace gravity by universal expansion of substance in virtue of EEP and previous remarks, and the absence of visual perception of expansion only prevents us to adopt it.

We need to examine one wonderful question arisen with Hubble's expansion, to evaluate the actual significance of our visual perceptions. The matter is there is no reasonable answer to the question; why does universe expand within global scale and is it unchangeable in short-local scale? The question is lawful since the same common laws of nature determine behavior of material objects independent from scales ${ }^{9}$. Then we can simply state, judging from the circumstances of the problem.

The image of expanded universe was created using different methods of evaluations.

The expansion of universe in large scales was accepted in virtue of Doppler shift of light frequency, meanwhile we judge about unchangeable sizes of our planetary system as well as galaxy in virtue of direct visual observations. We have no technical opportunities to visual observe of geometrical changes in large scale, for the faraway cosmic objects, and we adopt their motion in virtue of light's frequency change only. The frequency changes, however, are peculiar to the local scale cosmic systems also that are visually seen unchangeable (red shift of Sunlight, reaching to Earth, for example). The deepest subjectivity of our methodology becomes obvious from above-said. We observe the frequency change on distance as a common peculiarity of universe, in fact. Thus, we adopt the frequency change as evidence of movement and expansion in large scales, where we are unable to observe visually. However, we explain the same results of observations as an unexplainable to us "gravity influence", in scales suitable for our visual observations. Thus, we are facing the inevitable choice:

-We must adopt the observed cosmic expansion as common-universal, attributing it to material substance also, sacrificing our intuition, or:

-We must accept a large group of known facts as a chain of incredible coincidences.

The second one was accepted, in fact, due to huge pressure of human intuition. We prefer the first one however, considering the known role of mentioned factor in science history in general and the harmony of world perception that opens up with this. Then this conception becomes well confirmed with dominant conviction of creation of Universe (Big Bang Theory) and it provides additional evidence on the issue. It simply says that all cosmic objects, which we now see in gravitational balanced condition, were created from an insignificant "small" space and from one single kind of proto substance. Otherwise, the harmonically-proportional expansion of our material world will be impossible, since independent kinds of realities, having the same, similar-equal peculiarities of expansion, seem extremely improbable. By presented judgments, we come to an important conclusion on the single kind of physical reality being the basis of the substance, creating all possible material things. Thus, the next natural question arises: what kind of reality may serve the basis of all? Einstein was deeply convinced; "the electromagnetic field is enough for that" and he stubbornly worked on the idea, until the end

\footnotetext{
${ }^{9}$ Here we meant verbal character and actual absence of significance the restriction of locality in EEP.
} 
of his days (about 30 years), he did not succeed to complete it, however, mostly, because of non-comprehension of the causal essence of gravity as in Ref. [8].

The principal possibility of realization of Einstein's fundamental idea, on a single kind of primordial physical reality, is shown with representation of known elementary particles and their interactions based on the electromagnetic field as in Refs. [1] [4].

It is also possible to mark some observed results in favor of the presented concept. Particularly, the trajectory deflection of NACA's spacecraft "Pioneer" in correspondence to Hubble's constant is possible to observe as non coincidental, as some researchers are inclined to think as Ref. [28]. Meanwhile, it can be explained with actual expansion of our Solar system, in accordance to Hubble's universal expansion, with expansion of the material substance in general (that remains invisible to us).

The resent observations of concentrically expanding groups of cosmic objects also may serve as next serious evidence on the expansion concept in general, as in Ref. [29]. We see it appropriate referring to a recent publication directly evidencing the rightness of the developed concept of universality of expansion, as Ref. [30].

It is possible to comprehend that universal expansion of the world, consisting of manifold single kind objects will be unobservable (indescribable) within framework of idealized abstract mathematical concepts, without consideration and study of natural properties and peculiarities of physical objects. It means, in complete description of gravity phenomena a couple of known basic natural constants $c, h$ must be expressed. Einstein's GR (and other theories) give us quantitative descriptions of gravity, without causal connection of it to the basic particles of substance that necessary to complete the theory.

\subsection{Some Quantitative Reasoning on Universal Expansion}

We have no intention to put under doubt and challenge the quantitative significance of GR in general, in conformity to previous content. Meanwhile some clarifications, possible simplifications and important conclusions can be easily derived, as we know the causal base of studied phenomena, as we believe. These can be suitable for experimental test.

a) The complicated math apparatus of GR is easy to explain since it relates to "distorted-observable" values and events, and not to "actual events and laws of nature" (1.1. f)), (2.1.5). A second complication with GR is related to universal character of phenomena where "everything" participates in expansion process and all physical values and units become someway-interconnected variables. We can judge from (3.9) that there will be no way to see (measure) the geometrical changes of the expansion process in case of idealized-instant measurements (if we accept $c \rightarrow \infty$ ) in virtue of proportional changes of any real-physical etalons and observation systems in our world. Einstein's equations as well as Schwarzschild solution simply evidence the above-said (3.5), (3.4); all kinds of relative effects become zero if we accept the light velocity as infinite. We will detect action forces between contacting bodies and see "free falling" etc. that will be unexplainable to us, as actually seen.

Some secondary consequences of expansion process only may be detectable by direct vision that becomes possible thanks to limited light velocity.

We see the "far" events, connected to the motion with some delayed time, therefore certain difference relative to events "close" to us can be observed. The light velocity and corresponding parameters of material objects define the significance of "distortion" related to universal expansion that will be arguments in our formulas. Above-said may serve as a causal essence of GR effects in general.

We can illustrate one serious critical remark addressed to GR that puts it under doubt in general, based on above-said, as example. The matter is some theorists have observed that:

GR is not adequate from energetic point of view, since the gravitational energy gets different significances depending on the choice of observation systems, as in Ref. [7].

We can comprehend the essence of the problem considering that GR relates to observable values and events, and not to actual ones as said above. Then it becomes clear that-GR is not suitable for causal description of phenomena at all. We must pass into idealized thought system of observation, with absolute constant units of measurement to have the actual picture of gravity phenomena that will correspond to their casual mechanisms, similar to Copernicus' logical operations (1.1. c)). We already used the same operation to explain SR phenomena causally as observable distortions of reality (previous chapter).

The problem, however, becomes technically complicated in this case, because we need to consider new relations, arising due to dependencies of examined physical values on properties and parameters of material substance that we need to consider. 
b) We will use the local systems of observation, which is maximally comfortable, to simplify our calculus, in comparison to covariant description that used in GR. Moreover, we will examine particular cases only: the homogeneous symmetrical distribution of material substance and the absence of axial-angular momentum also. In this way, we can apply the single coordinate description that changes nothing from conceptual point of view and extremely simplifies the work.

c) Initially we intuitively have supposed in the classic physics the opportunity for us to mark absolute static systems and sizes (3.2. f)) and the unchangeable course of time with evenly standard intervals. We have never thought to link our units of measurements with concrete material objects, as we have assume these unchangeable at all, therefore it has not been important how those were set in practice. We can immediately understand now that our "meter" will change proportionally together with all material objects.

However, the question with the "time" is not so easy to solve as we do not have yet an unambiguous definition: what is meant under the term "time" at all!

Thus, we are just obligated to ask the natural question: what will happen with our clocks with universal expansion? For this, we must first answer the question, how is "time" linked with material substance? We have used in practice some real physical objects as the etalons of clocks that are able to generate some repeatedly-regular events, the frequency $f$ of which we can adopt as the time course or its inversely-propositional value as the time interval: $t=1 / f$. We can construct our clock from simplest form of substance-ideal gas. It is possible to conclude that events' frequency in standard condition (for example, the collision of two molecules of gas in certain volume) will be proportional to their distribution density:

$$
f \propto \rho \text { and: } t=1 / f \propto 1 / \rho
$$

We adopt (3.10) as definition of "realistic time" connected with substance and variable with the expansion, vs. abstract concept of "time" in supposition of it as unchangeable-absolute. We need to adopt proportionally symmetrical expansion of sizes to all possible material objects that we perceive as "unchangeable" as well:

$$
V \propto R
$$

where: $V$ velocity of expansion and $R$ is the distance.

The light velocity serves as an important factor that gives us possibility to observe certain secondary effects connected to universal expansion (the GR effects, in analogy to SR effects). Thus, we need to adopt restricted speed of our possible measurements also:

$$
V_{m s}=c<\infty
$$

where: $V_{m s}$ maximum velocity of measurements (observations).

Thus, (3.10), (3.11), (3.12) are the basic principles to describe the expanding world (the consequences of which we perceive as "gravity phenomena").

Let us suppose the expanded substance distributed homogenously by density $\rho$, in the spherical body with radius $R$ (Figure 5).

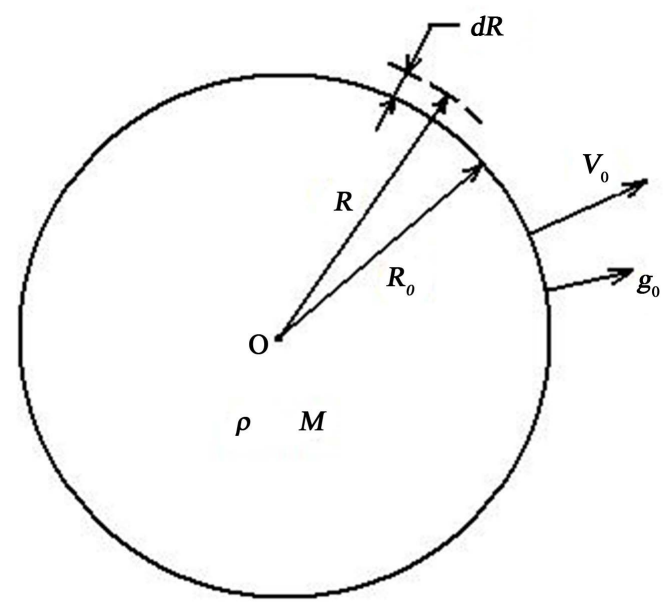

Figure 5. The expansion of material body. 
We can write from (3.10)

$$
\rho=3 M / 4 \pi R^{3}, t=k \frac{R^{3}}{M} \text { and } \mathrm{d} t=\frac{3 k}{M} R^{2} \mathrm{~d} R
$$

where: $M$ the mass; $k$ certain coefficient. We can write from (3.11) and from Figure 5.

$$
V=\frac{V_{0}}{R_{0}} R \text { and } \mathrm{d} V=\frac{V_{0}}{R_{0}} \mathrm{~d} R
$$

We define the acceleration of surface point relative to center in radial direction:

$$
a=\frac{\mathrm{d} V}{\mathrm{~d} t}=\frac{V_{0}}{3 k R_{0}} \cdot \frac{M}{R^{2}}
$$

where: $V_{0}, R_{0}$ velocity of point on the surface and its radius.

We adopt $m_{i}=m_{g}=m(3.8)$ and $a=g_{0}$ because of identity inertia and gravity (3.2. f)) and we get from (3.15) the Newton's law of gravity:

$$
F_{g}=\frac{\mathrm{d} V}{\mathrm{~d} t} m=\frac{V_{0}}{3 k R_{0}} \cdot \frac{M m}{R^{2}}=\frac{G M m}{R^{2}}
$$

where: $\frac{V_{0}}{3 k R_{0}}=G$ is the Cavendish constant established experimentally.

We can define the significance $V_{0}$ as the final speed in the end of way $R$ from known formula of accelerated movement, accepting $R=R_{0}$ :

$$
V_{0}=\sqrt{2 g_{0} R_{0}}=\sqrt{\frac{2 G M}{R_{0}}}=V^{e}
$$

where $V^{e}$ called the escape velocity known within frame of Newton's gravity. We see then $V_{0}$ corresponds to $v_{g}$ in (3.4): $V_{0}=V^{e}=v_{g}$.

We can write from (3.17), using the density instead of mass:

$$
V_{0}=R_{0} \sqrt{\frac{8 \pi G \rho}{3}} \text { and } \rho=\frac{V_{0}^{2}}{R_{0}^{2}} \cdot \frac{3}{8 \pi G}
$$

We can then define $k$ and $t$ from (3.13), (3.15)

$$
k=V_{0} / 3 G R_{0} \text { and } t=R_{0} / 1.5 V_{0} .
$$

The significance of $t$ in (3.19) corresponds to virtual time that needs to pass from initial point of expansion ( 0 ) to surface, calculated by present scales and units of measurements. The expression (3.19) shows relative meaning of time depending on parameters of material objects. The local character of "time" as property of a concrete material object and wrongness of operation with "universal time" concept become clear by the same.

We emphasize that our definition of "time" (3.10) corresponds to components of stress-energy tensor $\boldsymbol{T}_{\boldsymbol{\mu}}$ in Einstein's Equations (3.5) by using known mass-energy relation $E=m c^{2}$ :

$$
f \propto \rho \propto \rho_{e}
$$

where $\rho_{e}$ is the density of energy.

(3.20) shows that the concept of "time" characterizes the energetic condition of substance. Thus, "time" can have only local-concrete meaning and not abstract universal, separate from material objects that are silently accepted in classic physics. This definition of physical "time" directly corresponds to Einstein's realistic demand (1.1. f)). The relation (3.18) by its form and members corresponds to Hubble's law. We accept the expansion of substance identical to Hubble's expansion (3.2.3)):

$V_{0} / R_{0} \equiv H$, and we get from (3.18):

$$
\rho=\frac{3 H^{2}}{8 \pi G}
$$


The observable closeness $\rho \rightarrow \rho_{c r}$ may evidence the rightness of this identification.

Then the addition of "balancing" constant $\Lambda$ to Einstein's Equation (3.5) becomes unnecessary, since Hubble's expansion and gravity become the same factor, interconnected and equal (as action with reaction in Newton's 3rd law). We can conclude also that mystically "dark matter" and "dark energy" seems as unnecessary, and the corresponding observable phenomena must be explained in the frame of outlined concept (will discussed).

d) To define relativistic effects connected with universal expansion (gravity) we need to consider 3rd principle (3.12). The interconnected factors of expansion speed and acceleration, in combination with light velocity will be the arguments defining this or that observable relativistic effects, as it derives from above content. We will study the significance of two mentioned factors separately due to comfort of their application for the concrete effects.

Let's assume, an observer measured the distance $R_{0}$ using the light signal passing from center to surface (Figure 5). The measured time will be $R_{0} / c$ without consideration of expansion. There will be certain delay of time because of expansion speed:

$$
\Delta t=\frac{R_{0}}{c-V_{0}}-\frac{R_{0}}{c}=\frac{R_{0} V_{0}}{c^{2}-c V_{0}}
$$

We define the relative increase of measured value using (3.22):

$$
k_{v}=\frac{\Delta R}{R_{0}}=\frac{\Delta t V_{0}}{R_{0}}=\frac{V_{0}^{2}}{c^{2}-c V_{0}}=\frac{V_{0}^{2}}{c^{2}\left(1-V_{0} / c\right)}=\frac{V_{0}^{2}}{c^{2}}+\frac{V_{0}^{3}}{c^{3}}+\frac{V_{0}^{4}}{c^{4}}+\cdots+\frac{V_{0}^{n}}{c^{n}}
$$

Using (3.17), we get:

$$
k_{v}=\frac{2 G M}{c^{2} R_{0}}+\left(\frac{2 G M}{c^{2} R_{0}}\right)^{\frac{3}{2}}+\frac{4 G^{2} M^{2}}{c^{4} R_{0}^{2}}+\cdots+\left(\frac{2 G M}{c^{2} R_{0}}\right)^{\frac{1+n}{2}}
$$

We will be satisfied by examination of "weak" gravity, accepting $V_{0} \ll c$ that brings to:

$$
k_{v} \approx \frac{2 G M}{c^{2} R_{0}}
$$

The result (3.25) may serve as illustration of physical meaning of Schwarzschild solution and corresponding "curvature of space-time" (3.4). Meantime, we see that (3.24) provides additional correcting members despite incomparable simplicity of used considerations.

We need to consider the increase of expansion speed also during the measurement that will be defined by acceleration factor. The increase of expansion speed will be:

$$
\begin{gathered}
\Delta V_{g}=g_{0} \frac{R_{0}}{c} \text {, and } k_{g}=\frac{\Delta V_{g}}{c}+\frac{\left(\Delta V_{g}\right)^{2}}{c^{2}}+\cdots+\frac{\left(\Delta V_{g}\right)^{n}}{c^{n}} \text {. Using } g_{0}=\frac{V_{0}^{2}}{2 R_{0}} \text { from (3.17) we get: } \\
k_{g} \approx \frac{G M}{c^{2} R_{0}}=0.5 k_{v}
\end{gathered}
$$

We bring causal illustrations to some known GR effects by using deduced factors $k_{v}, k_{g}$.

\section{- "Gravity influence" on the frequency of light}

Let us assume light's signal passes way $l \ll R_{0}$ near to surface of material body in radial direction. Doppler frequency change will appear in consequence of expansion. Own expansion of $l$ may be ignored in virtue of initial condition and the frequency change will be defined mostly with the acceleration factor.

$$
\mathrm{d} f / f \approx \mathrm{d} V / c=g_{R} \mathrm{~d} R / c^{2}=G M \mathrm{~d} R / c^{2} R^{2}
$$

The summary effect on two opposite radial directions will be defined:

$$
\Delta f / f \approx \pm\left(G M / c^{2}\right) \int_{R_{0}}^{R_{0}+l} \mathrm{~d} R / R^{2}=\mp \frac{G M}{c^{2} R_{0}} \cdot \frac{l}{R_{0}+l_{0}}=\mp k_{g} \cdot \frac{l}{R_{0}+l}
$$

The same effect is interpreted with GR as "consequence of difference of gravity potentials": 


$$
\Delta f / f= \pm \Delta \phi / c^{2}=\mp \frac{G M}{c^{2}}\left(\frac{1}{R_{0}}-\frac{1}{R_{0}+l}\right)=\mp k_{g} \frac{l}{R_{0}+l}
$$

(This effect has been confirmed repeatedly, for example, by Shapiro in laboratory and later by NASA in cosmic scale (Gravity Probe-A).

\section{- Deviation of light ray near massive body}

The light's ray looks curved in consequence of universal expansion. The massive body $\boldsymbol{M}$ expands from dashed line to solid, accordingly, the observer's position changes during light passage from the edge of material body to the observer (Figure 6). The position of light source seems shifted with angle $a$. The factors of speed and acceleration of expansion participate in the phenomenon in virtue of scales. We can define the share of expansion speed immediately considering that it is perpendicular to light's ray as bellow:

$$
a_{v} \approx \operatorname{tg} a_{v}=k_{v}
$$

The component of acceleration $g_{n}$ perpendicular to ray only causes the curvature, which is changeable along the way. We introduce variable coordinate $x$ to define $g_{n}$ (Figure 6).

$$
g_{n}=g_{R} \frac{R_{0}}{R}=\frac{G M R_{0}}{R^{3}}=\frac{G M R_{0}}{\left(x^{2}+R_{0}^{2}\right)^{3 / 2}}
$$

While the ray passes the elementary distance $\mathrm{d} x$ the expansion speed will increase in perpendicular direction: $\mathrm{d} V_{n}=g_{n} \mathrm{~d} x / c$. We define the summary change of speed accepting that the light's ray passes the distance $(-\infty \rightarrow+\infty)$.

$$
\Delta V_{n}=G M R_{0} \int_{-\infty}^{+\infty} \frac{\mathrm{d} x}{c\left(x^{2}+R_{0}^{2}\right)^{3 / 2}}=\frac{2 G M}{c R_{0}} \text { and } a_{g} \approx \operatorname{tg} a_{g}=\frac{\Delta V_{n}}{c}=\frac{2 G M}{c^{2} R_{0}}=2 k_{g}=k_{v}
$$

The resulting deviation angle will be:

$$
a=a_{v}+a_{g} \approx 2 k_{v}=4 G M / c^{2} R_{0}
$$

It coincides with prediction of GR that the first time was confirmed by Eddington in 1919.

\section{- The angular displacement of planets' orbits}

Causal interpretation of the phenomenon is the same as in above-examined effects. Two factors $k_{v}, k_{g}$ participate in the effect as the observer's location is supposed to be connected to the central body which extremely simplifies the calculations.

The relative expansion of orbit's radius during observation will be: $\Delta R / R=k_{v}+k_{g}=1.5 k_{v}$

The expansion of orbital length will be $\Delta L=2 \pi \Delta R$ and angular displacement will be:

$$
\Delta \varphi=\Delta L / R=2 \pi \Delta R / R=3 \pi k_{v}
$$

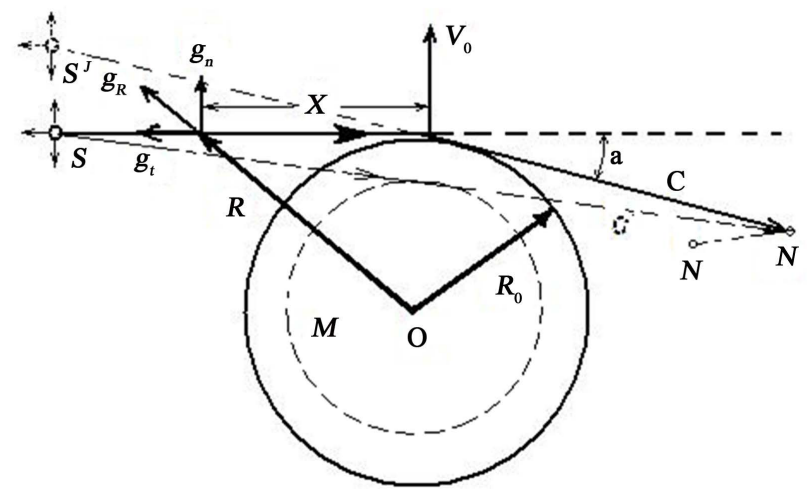

Figure 6. Light rays deviation near massive body. $\boldsymbol{M}, \boldsymbol{R}_{\mathbf{0}}$ : massive body; $\boldsymbol{S}$ : light source; $\boldsymbol{N}$ : observer's location; C: light ray; a: deviation angle. 
We use $a(1-e)$ instead of $R$, where $a$ is the long axis and $e$ is the elliptic parameter of orbit, and we get the final expression of angular change as:

$$
\Delta \varphi=\frac{3 \pi k_{v}}{a(1-e)}=\frac{6 \pi G M}{c^{2} a(1-e)}
$$

This result was confirmed first time in relation to the planet Mercury that was resolute for recognition of the whole significance of GR.

\section{- The light signal's "delay in gravity field"}

This prediction of GR also was confirmed by different observations. Meanwhile, one undesirable conclusion follows from a phenomenon that is unspoken. The matter is the light signal "slows down" in case if it passes vs. gravity field (light radiates from central body). The effect gets the opposite sign however, in opposite direction by the same formulas. Then the velocity of light exceeds $c$ that looks contradicting to basic principle of SR! The problem is simple to explain within the expansion concept; the passed way of light's signal $l$ increases (decreases) during the observation (measuring) in consequence of expansion, and the light's velocity does not change. We shall define the time delay considering variability of factors $k_{v}, k_{g}$ in scales of observation $l \gg R_{0}$.

$$
\mathrm{d} t=\left(k_{v}+k_{g}\right) \frac{\mathrm{d} R}{c}=\frac{3 G M}{c^{3}} \cdot \frac{\mathrm{d} R}{R} \text { and we get: } \Delta t= \pm \frac{3 G M}{c^{3}} \int_{R_{0}}^{R_{0}+l} \frac{\mathrm{d} R}{R}= \pm \frac{3 G M}{c^{3}} \ln \frac{l}{R_{0}}
$$

Similar experiments are also realized by NASA. We shall remark an important point on above examples that all results are based on the same causal concept which may serve as an evidence of its correctness. In author's view:

-The possibility of unification of Newton's gravity, Hubble's expansion and Einstein's GR in the same causal context may be adopted as weighty evidence on significance of the offered concept.

The interpretation of other relativistic effects with the same comprehensible causal basis also seems possible. Particularly, the effects connected with presence of angular momentum can be calculated by consideration of the Coriolis Effect arising with expansion process (as Lense-Thirring effect etc.).

e) Some "obvious" problems with universal expansion concept arise that need to be examined.

\section{- The absence of "gravity waves" follows from offered concept}

The question however has following simplest explanation: "gravity waves" have arisen by verbal-arbitrary interpretation of GR, which was illustrated in the previous content.

Einstein's Equations (3.5) are adopted to present the "field equations" and existence of its "indignant states" as well as "gravity radiation" follows formally. The absence of influence on distance, however, is one of the main principles of GR that brought to correct results. Then some internal contradiction arises between "gravity waves" (as the "influence on distance") and "movement by geodetic, free of influence" (the verbal replacement of "field" with the "curvature of space-time" does not remove the problem).

Meantime, it has shown already that GR presents by itself a quantitative description of expanded world and Einstein's equations actually describe the observable movement exclusively. Thus, "gravity wave" becomes a result of misinterpretation of used concepts. The same conclusion derives from direct identification of "gravity" with the "inertia" that is actually adopted in GR (as well as in Newton's gravity). Then it becomes simply obvious that "the inertial waves passing a distance" cannot exist by definition, as the inertial force influence is transferred through direct contact of material objects only. It becomes clear from above-said that experimental detection of "gravity wave" means violation of identity of concepts of gravity and inertia that will be crucial for the offered concept in whole. The experimental confirmation of non-distinguishable gravity and inertia, however, seems easier realizable to us (3.2. f)) than "gravity wave detection" which may remove this problem from the agenda at all, as artificially created. We hope the experimenters may consider the above-said as obvious argumentations and such experiments may be implemented.

- Problems with "dark matter" and "dark energy"

One of the aspects of introduction of "dark matter" was connected to the Hubble's expansion and to the cosmological member, that was examined above (3.3. b), c), d)). Some observed results, however, have pushed to recover the "dark matter" in addition to "dark energy" as well. The issue is, certain cosmic systems have been observed, where significance of Newton's gravity looks as exceeded, while evaluating the quantity of gravitating substance by known criterions. Then theorists introduced some unclear kind of "reality" necessary to com- 
pensate the "deficit" of gravity. This approach cannot be acceptable to us in virtue of adopted methodology as a pure "ad hoc" hypothesis without any evidence of its existence. Meanwhile, the concept of expansion opens clear opportunity to solve similar cognitive complications as consequences of differences between observable and actual pictures of reality (3.3. a)) (i.e. similar to SR problems). Different "deviations from known natural laws" and causal paradoxes may be observed then that may be pushing to introduce a new hypothetical reality. For example, some increase of planet's orbital movement (3rd point, in 3.3. d)) may be interpreted as "some increase of gravity field" of central body, if desired. The decrease of orbital movement is also possible to observe depending on parameters and observation systems that will demand additional "repulsive forces" with their corresponding sources etc. Then the essence and actual significance of examined problems become clear. We need to notice, that some researchers do not share modern hypotheses on "dark matter"/“energy" and have disputed this approach with certain arguments as in Ref. [31].

\section{- Problems with orbital movement and heavenly mechanics}

The examined identity of concepts of "gravity" and "inertia" are enough to present the same consequences of gravity as phenomena connected with the universal expansion of substance as our equations remain the same (Newton's gravity as well as GR). However, a huge number of immediate questions beg there with "switch" from gravitation to inertia. The situation is similar to intuitive reaction of the announcement of the Earth as something round, on its movement etc. We will look at couple of probable questions only:

-The Moon "feels" through gravity field where the Earth is, and "may choose" the path to move around it. How can it "understand" now: where is the Earth's location? The answer is easy to find considering that expansion is accelerative that is characterized by three physical parameters: direction, speed and acceleration relative to a free, uniform movement that contains in it two parameters only (direction and speed). It follows that the body that is expanding with acceleration, "remembers" the initial point where it has started the process. It will do corresponding oscillations around it by getting certain external impulse. The explanation that the Earth and the Moon have consisted in one common body in the time follows from this. They have been divided by some scenario; both of them now oscillate around the initial common point of their masses, under initial equal impulses on opposite directions.

\section{- Next problem concerns the "obvious" conclusion of infinite increase of expansion speed with time due to its accelerating character}

A quantitative explanation of the problem is also possible, despite that it sounds somewhat unusual in the framework of adopted traditional concepts. The question is related to "time" concept that was the "universal-abstract" before and now it is directly defined with the density of mass-energy (3.10), (3.20). The inverse proportionality of unit interval of time to density of substance brings to permanent increase of next interval of time in relation to the previous. Thus, permanent increase of speed (the first differential of distance by time) arises as result of decrease of regular events' frequency due to universal expansion. It simply follows from relations (3.13), (3.14), (3.15). The "actual picture" of expansion (that we would see in imaginary absolute system, with unchangeable timers and meters) would be seen by exponential law, where the final speed of expansion strives to the light velocity: $V \rightarrow c<\infty$, with $\tau \rightarrow \infty$. i.e., a wrong conclusion arises because we observe a distorted picture of expansion (except Hubble's expansion that we "see" indirectly). We bring some simplest considerations. Using (3.14) and accepting "time" as an independently free variable, we write:

$$
\begin{aligned}
& \frac{\mathrm{d} R}{\mathrm{~d} t}=R \frac{V_{0}}{R_{0}} \text {. And we get } \ln \frac{R}{R_{0}}=\frac{V_{0}}{R_{0}}\left(t-t_{0}\right) \text {. Accepting } t_{0}=0 \text { we get: } \\
& R=R_{0} \mathrm{e}^{V_{0} t / R_{0}} \text {. Using (3.18), we get: } \\
& \qquad R=R_{0} \exp \left(\sqrt{\frac{8 \pi G \rho_{0}}{3}}\right) \cdot t \text { and: } t=\ln \left(R / R_{0}\right) \sqrt{\frac{3}{8 \pi G \rho_{0}}}
\end{aligned}
$$

The time change with expansion in imaginary absolute system is illustrated (Figure 7).

\subsection{Energy of Expansion: Gravity Constant}

The gravitational energy is connected to expansion speed, as it follows from the offered concept. It represents kinetic energy, concentrated exclusively in the expanded body. Then its dependence on the system of observation becomes simply explainable. 


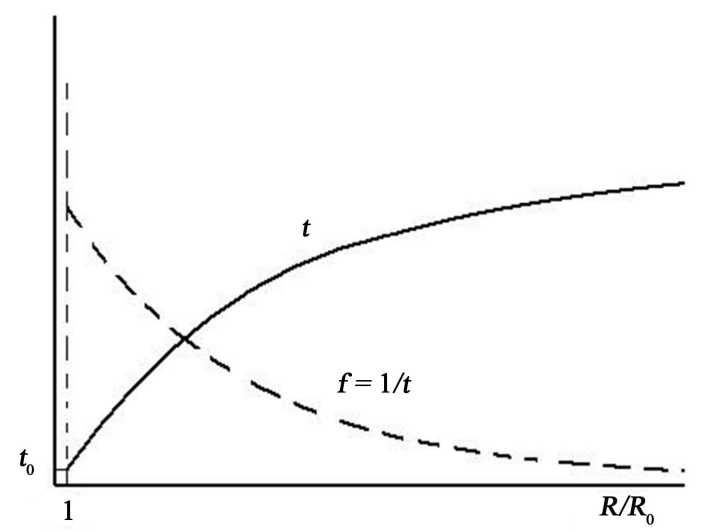

Figure 7. Time change with expansion.

a) We shall bring here one most important remark for following considerations. From above-said follows, that gravity characteristic of the substance itself has a local significance

It means the peculiarities of expansion are defined by the parameters of a concrete material object. It has been expressed in our formulas, where the adopted "gravity constant" always acts in combination with the massenergy density of substance (or, with "local time" (3.3. c)). Above-said means that "gravity" peculiarity of substance cannot be an independent fundamental constant of nature (in the rank of $h, \mathrm{c}$ ) and it may be defined within the dependence on certain free parameter of substance, in combination with $h, c(3.2$. g), 2)). Thus, we must adopt that the experimentally established known to us "gravity constant" becomes variable in the imaginary absolute system, parallel to course of time, with our length units etc. ${ }^{10}$. It becomes clear also, that adopted "gravity constant" is not "so successful" in virtue of possibility of simplest definition, following from (3.14). The relation $V / R$ becomes a sort of local constant for a concrete material object in absolute system of observation, due to the demand of symmetry preservation during expansion (that we observed as Hubble's expansion). Thus, we examine relation $V=f(R)$ for concrete material object to open causal essence of expansion. We shall define kinetic energy of expansion for the standard body $M, R_{0}$ relative to its center (Figure 8).

The kinetic energy corresponding to elementary volume between $R, R+\mathrm{d} R$ will be:

$\mathrm{d} w=V^{2} \mathrm{~d} m / 2$, where: $V=V_{0} R / R_{0} \quad$ (3.14), $\mathrm{d} m=4 \pi R^{2} \rho \mathrm{d} R=3 M R^{2} \mathrm{~d} R / R_{0}^{3} \quad$ and we get:

$$
w=\int_{0}^{R_{0}} \mathrm{~d} w=\int_{0}^{R_{0}} \frac{3 M V_{0}^{2}}{2 R_{0}^{5}} R^{4} \mathrm{~d} R=\frac{3}{10} M V_{0}^{2}
$$

Using (3.17), we can write:

$$
w=3 G M^{2} / 5 R_{0}
$$

(3.34) appears equal to full "energy of gravitational field" that is calculated by the next imaginary operation: the "gravity source" disintegrates to elementary parts that are shifting away on infinite distance. The resulting energy that needs to be spent to overcome "attraction forces" to complete the described operation gives the same result. Thus, the exact coincidence of results of two considerations confirms the full identity of concepts "gravity" and "inertia" by their energetic significances also (i.e. without "local" restriction).

b) Derivation of the gravity constant: The universal, proportional expansion of material world may be possible in case of a single kind of basic substance, creating all possible kinds of elementary particles, localized (such electron, proton, etc.) as well as non-localized (photon) (3.2. g). 3)). The reader can find physical models of elementary particles as localized and non-localized quanta of electromagnetic fields in Refs. [1] [4]. Then we can conclude that energy (or velocity) of expansion may have only electromagnetic nature, as it follows from this presentation. We can simply suppose that expansion must be connected to the electromagnetic coupling constant, i.e., it will be defined by the fundamental fine structure constant $(a \approx 1 / 137)$ as well as all other kinds of interactions of particles and all known physical-chemical peculiarities of substance in general as it

\footnotetext{
${ }^{10}$ We mark that above-said may serve as another explanation to observable difference of gravity constant, depending on distance and density of substance, thus, to a necessity introduction of "dark matter-energy".
} 


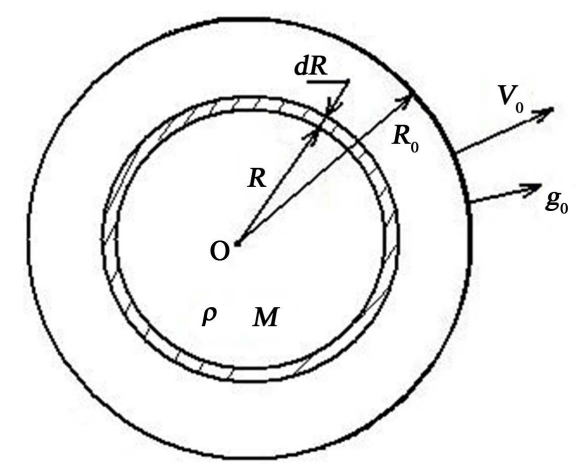

Figure 8. The energy of expansion.

presented in Ref. [32]. We test the supposition with electron's physical model as localized Compton wave polarized circularly, in view of its simple structure (as wave interference-standing wave) relative to other particles Refs. [1] [4]. We will choose certain system of description adopting: $[L] \equiv \lambda_{e} \equiv c \equiv 1$ where: $\lambda_{e}$ is the Compton's wavelength for the electron). We write our basic supposition in chosen relative units system in following simplest form:

$$
V_{e}^{*} \equiv a
$$

(3.35) corresponds to escape velocity of electron taking it as the "gravity source".

It may be transferred into real units system using below expression:

$$
V_{e}=a c\left|\lambda_{e} / c\right|
$$

where: $c$ serves as the velocity unit, and the numeric constant $\left|\lambda_{e} / c\right|$ taking into account the difference of local time units in two systems ${ }^{11}$ (3.3. c)).

We are using quantum relation (3.9) to define mass of the electron (according to adopted electron model in Ref. [1]).

$$
M=m_{e}=h / c \lambda_{e},
$$

We define speed as (3.17) $V_{e}=\sqrt{2 G m_{e} / R_{e}}$ where: $R_{e}$ is the electron radius:

$$
R_{e}=\lambda_{e} / 2 \pi \text { (see in Ref. [1]). }
$$

We obtain from (3.36)

$$
G=\frac{a^{2} c^{3} \lambda_{e}^{2}}{4 \pi h} \cdot\left|\frac{\lambda_{e}}{c}\right|^{2}
$$

The standing wave and diameter of the particle have some increase in examined model, corresponding to its anomaly magnetic moment: $\left(\mu_{e} / \mu_{B} \approx \lambda_{r} / \lambda_{e} \approx 1.00115965 \cdots\right)$ where $\lambda_{r}$ is the real-average wavelength as in Ref. [1]. We get from (3.37) final expression considering mentioned correction $\left(\lambda_{r} / \lambda_{e} \approx \mu_{e} / \mu_{B}\right)$ :

$$
G_{T}=\frac{a^{2} c^{3} \lambda_{e}^{2}}{4 \pi h}\left(\frac{\mu_{e}}{\mu_{B}}\right)^{4} \cdot\left|\frac{\lambda_{e}}{c}\right|^{2}
$$

We got $G_{T} \cong 6.6755 \times 10^{-11}\left[\mathrm{M}^{3} \cdot \mathrm{kg}^{-1} \cdot \mathrm{s}^{-2}\right]$ that well corresponds to recent measurements.

\section{Conclusions and Discussions}

The derived formula for the theoretical significance of gravity constant (3.38) may be interpreted as coinciden-

\footnotetext{
${ }^{11}$ We shall remind that "time" concept has local character in GR in fact, due of its definition as the density of energy-impulse in Einstein Equations (3.5).
} 
tal, if taken separately. However, in authors' viewpoint, it is difficult to do in the whole context of approach, considering a number of similar "coincidences". Meanwhile, the opportunity of cause-effect, harmonious and self-consistent representation of material world on the unique basis of substance and on the common principles of nature, may be a weighty evidence of significance of the offered concept and used methodology.

The productivity of the approach proves the correctness of the wave-dynamical representation of elementary particles and of the microcosm in whole as Ref. [1] [4]. It confirms the convictions and demands of undeniable founders Einstein, de Broglie, Schrodinger, Planck and other coryphées of physics unfairly rejected by the majority.

Offered causal interpretation confirms and clarifies quantitative significance of relativity theories; meantime it demands an important revision of the adopted cognitive interpretations (or to replenish their absence). These become conceptually completed, "full-blooded" physical theories, with the same.

New opportunities may open to explain certain problems with cosmology. Particularly, complicated processes of "Gravity collapse" and "Big Bang" may have new aspects of description, remaining in the framework of the known natural laws, i.e. without referring to the mystical "Singularity".

\section{References}

[1] Kirakosyan, G. (2013) Rethinking the Formal Methodology (I): Wave-Vortex Essence of the Substance. Hadronic Journal, 36, 9-51.

[2] Kirakosyan, G. (2013) Physika: Krizis I Vozmojniy Proriv. LAP: LAMBERT Academic Publishing, Russian. (Russ)

[3] Brillouin, L.N. (1970) Relativity Reexamined. Academic Press, New York.

[4] Kirakosyan, G. (2011) Modeling the Electron as a Stable Quantum Wave-Vortex: Interpretation $a \approx 1 / 137$ as a Wave Constant. Hadronic Journal, 34, 337-349.

[5] Kuhn, T.S. (1962) The Structure of Scientific Revolutions. University of Chicago Press, Chicago.

[6] Smolin, L. (2006) The Trouble With Physics. Houghton Mifflin Harcourt, Boston.

[7] Lindsay, R.B. (1967) Arbitrariness in Physics. Physics Today, 20, 23. http://dx.doi.org/10.1063/1.3034055

[8] Suvorov, S.G. (1966) Einstein's Philosophical Views and Their Relation to His Physical Opinions. Advances in Physical Sciences, 8, 578-609. http://dx.doi.org/10.1070/PU1966v008n04ABEH002991

[9] Einstein, A. (1921) The Meaning of Relativity. Princeton University Press, Princeton.

[10] Einstein, A. (1905) Zur Elektrodynamik bewegter Körper. Annalen der Physik, 322, 891-921. http://dx.doi.org/10.1002/andp.19053221004

[11] Einstein, A. (1905) On the Electrodynamics of Moving Bodies. 30 June. http://www.fourmilab.ch/etexts/einstein/specrel/www/

[12] Marmite, P. (2012) The Collapse of the Lorentz Transformation. 2 July 2012. http://www.newtonphysics.on.ca/lorentz/index.html

[13] Hoyle, F. and Narlikar, J.V. (1964) A New Theory of Gravitation. The Royal Society Publishing, London. http://rspa.royalsocietypublishing.org/content/royprsa/282/1389/191.full.pdf

[14] Henri, P. (1913) The Theory of Le Sage, the Foundation of Science. Science Press, New York, 517-522.

[15] Blair, D.G. (2005) The Detection of Gravitational Waves. Cambridge University Press, Cambridge. http://arcturus.ligo.caltech.edu/docs/public/P/P900010-00.pdf

[16] Corda, C. (2014) Differential Geometry and Scalar Gravitational Waves. 14 January. http://export.arxiv.org/pdf/1401.2873

[17] Soklakov, A.N. (2001) Occam's Razor as a Formal Basis for a Physical Theory. 26 September 2001. http://arxiv.org/pdf/math-ph/0009007.pdf

[18] Saakyan, G.S. (1985) Prostranstvo-Vremya i Gravitatia. State University Press, Yerevan. (Russ)

[19] Shapiro, I.I., Counselman, C.C. and King, R.W. (1976) Verification of the Principle Equivalence for Massive Bodies. Physical Review Letters, 36, 555. http://dx.doi.org/10.1103/PhysRevLett.36.555

[20] Logunov, A.A. (2002) The Theory of Gravity. 4 October 2002. http://arxiv.org/pdf/gr-qc/0210005v2.pdf

[21] Roll, P.G., Krotkov, R. and Dicke, R.H. (1964) The Equivalence of Inertial and Passive Gravitational Mass. Annals of Physics, 26, 442-517. http://dx.doi.org/10.1016/0003-4916(64)90259-3

[22] Baessler, S., Heckel, E.G., Adelberger, J., Gundlach, H., Schmidt, U. and Swanson, H.E. (1999) Improved Test of the Equivalence Principle for Gravitational Self-Energy. Physical Review Letters, 83, 3585. 
http://dx.doi.org/10.1103/PhysRevLett.83.3585

[23] Dittus, H. and Lämmerzahi, C. (2005) Experimental Tests of the Equivalence Principle and Newton's Law in Space. AIP Conference Proceedings, 758, 95. http://scitation.aip.org/content/aip/proceeding/aipcp/10.1063/1.1900510

[24] Marmet, P. (1997) Einstein's Theory of Relativity Versus Classical Mechanics. University of Virginia, Charlottesville.

[25] Fock, B.A. (1961) Teoriya prastranstvo, vremeny i tyagoteniya. State Publishing of Physics-Math Literature, Moscow, 301-302. (Russ)

[26] (2011) The Accelerating Universe. 4 August. http://www.nobelprize.org/nobel_prizes/physics/laureates/2011/advanced-physicsprize2011.pdf

[27] Kiselev, V.V. (2003) Acceleration Parameter of the Universe Expansion and Cosmological Equation of State. http://cds.cern.ch/record/636340/files/0308135.pdf

[28] Rosales, J.L. (2002) The Pioneer's Acceleration Anomaly and Hubble's Constant. http://arxiv.org/pdf/gr-qc/0212019.pdf

[29] Gurzadyan, V.G. and Penrose, R. (2013) On CCC-Predicted Concentric Low-Variance Circles in the CMB Sky. http://arxiv.org/pdf/1302.5162.pdf

[30] Rasor, N.S. (2014) Metric Expansion of Space and the Apparent Speed of Light. Physics Essays, 27, $247-252$.

[31] Santilli, R.M. (2015) Representation of Galactic Dynamics via Isoshifts without Universe Expansion, Dark Matter and Dark Energy. American Journal of Modern Physics, 4, 26-43. http://dx.doi.org/10.11648/j.ajmp.20150402.11

[32] Kirakosyan, G. (2015) Deduction of Coupling Constant ( $a \approx 1 / 137)$ as a Wave Peculiarity: Possible Laboratory Confirmation. Physics Essays, 28, 283-285. 